\title{
Implementation and benchmark of a long-range corrected functional in the density functional based tight-binding method
}

\author{
V. Lutsker ${ }^{a}$, B. Aradi ${ }^{b}$, and Thomas A. Niehaus ${ }^{a *}$ \\ a Department of Theoretical Physics, University of Regensburg, 93040 Regensburg, \\ Germany \\ ${ }^{b}$ BCCMS, University of Bremen, 28359 Bremen, Germany
}

\begin{abstract}
Bridging the gap between first principles methods and empirical schemes, the density functional based tight-binding method (DFTB) has become a versatile tool in predictive atomistic simulations over the past years. One of the major restrictions of this method is the limitation to local or gradient corrected exchange-correlation functionals. This excludes the important class of hybrid or long-range corrected functionals, which are advantageous in thermochemistry, as well as in the computation of vibrational, photoelectron and optical spectra. The present work provides a detailed account of the implementation of DFTB for a long-range corrected functional in generalized Kohn-Sham theory. We apply the method to a set of organic molecules and compare ionization potentials and electron affinities with the original DFTB method and higher level theory. The new scheme cures the significant overpolarization in electric fields found for local DFTB, which parallels the functional dependence in first principles density functional theory (DFT). At the same time the computational savings with respect to full DFT calculations are not compromised as evidenced by numerical benchmark data.
\end{abstract}

Keywords: Density functional based tight-binding - DFTB · long-range corrected exchangecorrelation functionals - range separation - photoelectron spectroscopy - quasi-particle energies . static polarizability

\section{INTRODUCTION}

Density functional theory (DFT) is currently the de-facto standard in computational chemistry and condensed matter physics. 1 Due to algorithmic improvements and the availability of high performance

\footnotetext{
* Corresponding author: thomas.niehaus@ur.de
} 
computing architectures, simulations with several thousands of atoms are becoming possible. ${ }^{2 .}$ This development paves the way for in-silico combinatorial chemistry and materials science $\sqrt[3.4]{4}$

Notwithstanding, more approximate methods like the well established density functional based tight-binding method (DFTB) still have their merits. $\stackrel{5}{5}$ The search for global minima on complex potential energy surfaces or the need for accurate phase space sampling in molecular dynamics simulations are just two examples in this context On the methodological level, DFTB has been extended in various ways during the last years. Such developments include the inclusion of spin-orbit interactions and non-collinear spin,,$^{7}$ van-der-Waals corrections ${ }^{8}$ and hybrid QM/MM schemes, ${ }^{9} 10$ as well as the description of electronic excited states using the time-dependent formalism TD-DFTB. $11+14$

Surprisingly, one of the most trivial steps in a DFT calculation, the change of the employed exchange-correlation (XC) functional, is comparatively cumbersome in DFTB. This is because DFTB relies on precomputed matrix elements of the Hamiltonian that are transformed to the molecular frame by Slater-Koster rules. 15 In addition, a consistent set of transferable pair potentials must be created by combined DFT/DFTB calculations on reference structures. While these steps are labor-intensive but straightforward for local and semi-local XC functionals, additional complications arise for hybrid and long-range corrected (LC) functionals that involve a fraction of Hartree-Fock exchange. In this case even the validity of the Slater-Koster rules is far from obvious and the quality of typical approximations in the DFTB framework like the neglect of three-center and crystal field terms must be newly assessed.

In previous work, the formal foundations of DFTB with LC functionals were already outlined 16 In this contribution we provide details of an efficient implementation of these ideas and benchmark the accuracy of the new scheme. The interest in LC functionals arises due to their inherent reduction of the notorious self-interaction error in DFT. The resulting benefits of these functionals are well documented. $17-23$ They yield the correct asymptotic $-1 / r$ behavior of the generalized Kohn-Sham potential which allows for the extraction of accurate ionization potentials and electron affinities (i.e., quasi-particle energies) from a single $\mathrm{N}$-particle computation. In addition, the localization of the electron density in extended systems - the typical realm of DFTB applications - is strongly improved with respect to local functionals. We demonstrate that these desirable properties are also operational in the DFTB method which invokes additional approximations beyond the choice of the XC functional.

After a specification of the XC functional used in this work in Section 2.1, we briefly summarize the results obtained in Ref. 16 before we provide a detailed account of our implementation. Section 3 is devoted to the results of the new approach and contains a benchmark of fundamental gaps for a series of organic molecules. In addition we discuss the molecular response to electric fields (Section 3.2) and comment shortly on the benefits of the new method for biological systems in Section 3.3 . 


\section{THEORY}

\subsection{Choice of the exchange-correlation functional}

In this work we use a functional that is based on the work of Baer, Neuhauser and Livshits. ${ }^{24}$ [25 Baer and Neuhauser use the adiabatic connection with a descreened electron-electron interaction $v=[1-\exp (-\omega r)] / r$ and derive an approximate form of the exchange-correlation functional. This form depends only on the range-separation parameter $\omega$ and provides the correct long-range behaviour of the potential:

$$
E_{x c}=E_{x c}^{\omega, D F T}+E_{x}^{\omega, H F} .
$$

The second term is given by

$$
E_{x}^{\omega, H F}=-\sum_{i, j}^{N / 2} \iint^{\prime} \psi_{i}(\mathbf{r}) \psi_{j}(\mathbf{r}) \frac{1-e^{-\omega\left|\mathbf{r}-\mathbf{r}^{\prime}\right|}}{\left|\mathbf{r}-\mathbf{r}^{\prime}\right|} \psi_{i}\left(\mathbf{r}^{\prime}\right) \psi_{j}\left(\mathbf{r}^{\prime}\right) d \mathbf{r}^{\prime} d \mathbf{r},
$$

where Kohn-Sham molecular orbitals are denoted by $\psi_{i}$. The expression in Eq. 2 approaches the exact Hartree-Fock (HF) exchange energy for large inter-electronic distance and is responsible for the reduction of the self-interaction error.

The explicit form of the DFT exchange-correlation functional $E_{x c}^{\omega, D F T}$ has still to be approximated. Livshits and Baer suggested to use a correlation functional in the generalized-gradient approximation (GGA) together with short-range exchange in the local density approximation (LDA) according to

$$
E_{x c}^{\omega, D F T}=E_{c}^{G G A}+\alpha E_{x}^{\omega, L D A},
$$

with some empirically determined parameter $0 \leq \alpha \leq 1$. The parameter set $\{\alpha, \omega\}$ was subsequently optimized by fitting to thermochemical data. Similar empirical schemes are widely used to calibrate the whole plethora of different hybrid exchange-correlation functionals. $\left.{ }^{17]}[20] 26+28\right]$ The aim of this work, however, is a proof of concept and we are interested in the qualitative improvement over the traditional DFTB scheme with respect to the failures due to the delocalization problem. Because of this we do not attempt any fine-tuning of the employed functional in the present work and simply use the $\mathrm{PBE}^{29}$ correlation functional and $\alpha=1$ in Eq. 3 .

For the LDA short-range DFT functional $E_{x}^{\omega, L D A}$, the exchange energy of the homogeneous electron gas with Yukawa interaction is used $30+32$

$$
\begin{aligned}
E_{x}^{\omega, L D A}[\rho] & =-\frac{3}{4}\left(\frac{3}{\pi}\right)^{1 / 3} \int \rho^{4 / 3}(\mathbf{r}) P(a(\mathbf{r})) d \mathbf{r} \\
P(a) & =1-\frac{8}{3} a\left(\arctan \frac{1}{a}+\frac{1}{4}-\frac{a}{4}\left(a^{2}+3\right) \ln \left(1+\frac{1}{a^{2}}\right)\right) \\
a & =\frac{\omega}{2 k}, \quad k=\left(3 \pi^{2} \rho\right)^{1 / 3} .
\end{aligned}
$$




\begin{tabular}{lrrrrrr}
\hline \hline element & \multicolumn{3}{c}{$r_{0}^{\text {basis }}$} & \multicolumn{4}{c}{$r_{0}^{\text {density }}$} \\
\hline & $\mathrm{s}$ & $\mathrm{p}$ & $\mathrm{d}$ & $\mathrm{s}$ & $\mathrm{p}$ & $\mathrm{d}$ \\
\hline $\mathrm{H}$ & 3.0 & & & 2.5 & & \\
$\mathrm{C}$ & 2.7 & 2.7 & & 14.0 & 14.0 & \\
$\mathrm{~N}$ & 2.7 & 2.7 & 14.0 & 14.0 & \\
$\mathrm{O}$ & 2.3 & 2.3 & & 9.0 & 9.0 & \\
$\mathrm{~S}$ & 3.8 & 3.8 & 4.4 & 9.0 & 9.0 & 9.0 \\
\hline \hline
\end{tabular}

Table 1: The compression radii $\left[\mathrm{a}_{0}\right]$ for the elements used in this work.

Results with this choice of $x c$ functional will be termed LC-DFTB in the following.

\subsection{Total energy and Hamiltonian}

In this section we provide a brief overview of the LC-DFTB method, following Ref. 16, together with an account of our practical implementation. The method is based on the linear combination of atomic orbitals (LCAO), where the single particle molecular orbitals $\psi_{i}$ are expanded into a set of atom-centered basis functions $\left\{\phi_{\mu}\right\}$ :

$$
\psi_{i}(\mathbf{r})=\sum_{\mu} c_{\mu, i} \phi_{\mu}(\mathbf{r})
$$

As in the traditional DFTB method, a minimal valence-only basis set is obtained from pseudo-atomic DFT calculations with the additional confinement potential $V_{\text {conf }}(r)=\left(r / r_{0}\right)^{2}$. The pseudo-atoms are considered to be spherically symmetric by equally distributing electrons over degenerate valence shells. In this study the functional is long-range corrected and defined by Eqs. 1 to 6 . The compression radius $r_{0}$ is usually taken to be proportional to the covalent radius of the respective atomic species. In the whole scheme, there are two confinement radii per element: $r_{0}^{\text {basis }}$ amounts to the basis set compression and $r_{0}^{\text {density }}$ is used for the compression of the atomic densities in the initial guess. ${ }^{5}$ For LC-DFTB we use the same confinement radii as previously reported for the mio-1-1 set,, 33 which are summarized in Table 1. We note that the basis set for sulfur features additional polarization functions. 34

The non-interacting reduced density matrix for the closed-shell case is given by

$$
\rho\left(\mathbf{r}, \mathbf{r}^{\prime}\right)=2 \sum_{i=1}^{N / 2} \psi_{i}(\mathbf{r}) \psi_{i}\left(\mathbf{r}^{\prime}\right)=\sum_{\mu \nu} \underbrace{\left[2 \sum_{i=1}^{N / 2} n_{i} c_{\mu, i} c_{\nu, i}\right]}_{P_{\mu \nu}} \phi_{\mu}(\mathbf{r}) \phi_{\nu}\left(\mathbf{r}^{\prime}\right)
$$


which defines the density matrix $(\mathbf{P})_{\mu \nu}=P_{\mu \nu}$ in the atomic orbital (AO) representation.

With these notations and the particular choice of $E_{\mathrm{xc}}^{\omega, \mathrm{DFT}}$ we are ready to write the total energy of the closed-shell LC-DFT in the AO basis

$$
\begin{aligned}
E & =\sum_{\mu \nu} P_{\mu \nu} h_{\mu \nu}+\frac{1}{2} \sum_{\mu \nu \alpha \beta} P_{\mu \nu} P_{\alpha \beta}(\mu \nu \mid \alpha \beta) \\
& -\frac{1}{4} \sum_{\mu \nu \alpha \beta} P_{\mu \nu} P_{\alpha \beta}(\mu \alpha \mid \beta \nu)^{\mathrm{lr}}+E_{\mathrm{xc}}^{\omega, \mathrm{DFT}}[\rho],
\end{aligned}
$$

where single particle Hamiltonian $h_{\mu \nu}$ and two-electron repulsion integrals are given by

$$
\begin{aligned}
h_{\mu \nu} & =\int \phi_{\mu}(\mathbf{r})\left(-\frac{1}{2} \nabla^{2}+v^{\mathrm{ext}}(\mathbf{r})\right) \phi_{\nu}(\mathbf{r}) d \mathbf{r} \\
(\mu \nu \mid \alpha \beta) & =\iint \phi_{\mu}(\mathbf{r}) \phi_{\nu}(\mathbf{r}) \frac{1}{\left|\mathbf{r}-\mathbf{r}^{\prime}\right|} \phi_{\alpha}\left(\mathbf{r}^{\prime}\right) \phi_{\beta}\left(\mathbf{r}^{\prime}\right) d \mathbf{r}^{\prime} d \mathbf{r} \\
(\mu \nu \mid \alpha \beta)^{\mathrm{r}} & =\iint \phi_{\mu}(\mathbf{r}) \phi_{\nu}(\mathbf{r}) \frac{1-e^{-\omega\left|\mathbf{r}-\mathbf{r}^{\prime}\right|}}{\left|\mathbf{r}-\mathbf{r}^{\prime}\right|} \phi_{\alpha}\left(\mathbf{r}^{\prime}\right) \phi_{\beta}\left(\mathbf{r}^{\prime}\right) d \mathbf{r}^{\prime} d \mathbf{r}
\end{aligned}
$$

here $v^{\text {ext }}$ denotes the external potential. Next, the density matrix is decomposed as $\mathbf{P}=\mathbf{P}_{0}+\Delta \mathbf{P}$, with some reference density matrix or initial guess $\mathbf{P}_{0}$. Such a decomposition is common for direct SCF approaches where the Hamiltonian is constructed iteratively. ${ }^{35}$ We choose the reference density matrix to be the superposition of atomic density matrices $\mathbf{P}_{0}=\sum_{A} \mathbf{P}_{A}$, which are obtained from the previous atomic LC-DFT calculations for each element. This choice parallels on the one hand the protocol in standard DFTB, on the other hand it is widely used as the initial guess in quantum chemistry codes.

We now expand the local part of the exchange-correlation functional around the reference density matrix up to second order in $\Delta \mathbf{P}$ :

$$
\begin{aligned}
E_{\mathrm{xc}}^{\omega, \mathrm{DFT}}[\rho] & =E_{\mathrm{xc}}^{\omega, \mathrm{DFT}}\left[\rho_{0}\right]+\left.\int \frac{\delta E_{\mathrm{xc}}^{\omega, \mathrm{DFT}}}{\delta \rho(\mathbf{r})}\right|_{\rho_{0}} \delta \rho(\mathbf{r}) d \mathbf{r} \\
& +\left.\frac{1}{2} \int \frac{\delta^{2} E_{\mathrm{xc}}^{\omega, \mathrm{DFT}}}{\delta \rho(\mathbf{r}) \delta \rho\left(\mathbf{r}^{\prime}\right)}\right|_{\rho_{0}} \delta \rho(\mathbf{r}) \delta \rho\left(\mathbf{r}^{\prime}\right) d \mathbf{r} d \mathbf{r}^{\prime}+\mathcal{O}\left(\delta \rho^{3}\right) \\
& =E_{\mathrm{xc}}^{\omega, \mathrm{DFT}}\left[\rho_{0}\right]+\sum_{\mu \nu} \Delta P_{\mu \nu} v_{\mu \nu}^{\omega, \mathrm{xc}}\left[\rho_{0}\right] \\
& +\frac{1}{2} \sum_{\mu \nu \alpha \beta} \Delta P_{\mu \nu} \Delta P_{\alpha \beta} f_{\mu \nu \alpha \beta}^{\omega, \mathrm{xc}}\left[\rho_{0}\right]+\mathcal{O}\left(\delta \rho^{3}\right)
\end{aligned}
$$

First and second derivatives of the functional in the $\mathrm{AO}$ basis are denoted here as $v_{\mu \nu}^{\omega, \mathrm{xc}}$ and $f_{\mu \nu}^{\omega, \mathrm{xc}}$, respectively. Eq. 14 represents the first approximation in LC-DFTB, which amounts to linearization of 
the local exchange-correlation potential. Inserting this into the total energy expression and following the procedure in Ref. 33, we rearrange the terms such that

$$
\begin{aligned}
E & =\sum_{\mu \nu} P_{\mu \nu} H_{\mu \nu}^{0}+\frac{1}{2} \sum_{\mu \nu \alpha \beta} \Delta P_{\mu \nu} \Delta P_{\alpha \beta}\left[(\mu \nu \mid \alpha \beta)+f_{\mu \nu \alpha \beta}^{\omega, x \mathrm{c}}\left[\rho_{0}\right]\right] \\
& -\frac{1}{4} \sum_{\mu \nu \alpha \beta} \Delta P_{\mu \nu} \Delta P_{\alpha \beta}(\mu \alpha \mid \beta \nu)^{\mathrm{lr}}+E_{\text {rep }},
\end{aligned}
$$

where the last term, the repulsive energy $E_{\text {rep }}, \frac{5}{\sqrt[5]{6}}$ depends only on the reference density. The second approximation is to replace the repulsive energy by a sum of fast decaying pair potentials as it is the case in standard DFTB. 33 This is a reasonable approximation also in the present range-separated formalism as shown in Ref. 16.

The Hamiltonian $H_{\mu \nu}^{0}$, evaluated at the reference density

$$
\begin{aligned}
H_{\mu \nu}^{0} & =h_{\mu \nu}+\sum_{\alpha \beta} P_{\alpha \beta}^{0}(\mu \nu \mid \alpha \beta) \\
& -\frac{1}{2} \sum_{\alpha \beta} P_{\alpha \beta}^{0}(\mu \alpha \mid \beta \nu)^{\mathrm{lr}}+v_{\mu \nu}^{\omega, x \mathrm{c}}\left[\rho_{0}\right]
\end{aligned}
$$

is treated in the two-center approximation

$$
H_{\mu \nu}^{0}= \begin{cases}\epsilon_{\mu}^{\text {free }} & \mu=\nu \\ H_{\mu \nu}^{0}\left[\mathbf{P}_{A}+\mathbf{P}_{B}\right] & \mu \in A, \quad \nu \in B \\ 0 & \text { else, }\end{cases}
$$

where the density matrix of the dimer $\mathbf{P}_{A}+\mathbf{P}_{B}$ is constructed with the density compression radius $r_{0}^{\text {density }}$. As already mentioned, the basis functions $\phi_{\mu}$ stem from a LC-DFT calculation with the compression radius $r_{0}^{\text {basis }}$ and are used to construct both the zeroth-order Hamiltonian matrix elements $H_{\mu \nu}^{0}$ and the overlap matrix $S_{\mu \nu}=\int \phi_{\mu}(\mathbf{r}) \phi_{\nu}(\mathbf{r}) d \mathbf{r}$. Thanks to the two-center approximation, matrix elements for all possible geometries can be constructed from a small set of high-symmetry integrals according to Slater-Koster rules. ${ }^{15}$ These parameters are thus tabulated as a function of inter-atomic distance $R_{A B}=\left|\mathbf{R}_{A}-\mathbf{R}_{B}\right|$.

Next we approximate the two-electron integrals using the Mulliken approximation

$$
\phi_{\mu}(\mathbf{r}) \phi_{\nu}(\mathbf{r}) \approx \frac{1}{2} S_{\mu \nu}\left(\left|\phi_{\mu}(\mathbf{r})\right|^{2}+\left|\phi_{\nu}(\mathbf{r})\right|^{2}\right)
$$

This allows for reduction of four-center integrals by a sum of two-center integrals $\gamma_{\mu \nu}$

$$
\begin{aligned}
(\mu \nu \mid \alpha \beta)^{\mathrm{lr}} & \approx \frac{1}{4} S_{\mu \nu} S_{\alpha \beta}\left[(\mu \mu \mid \alpha \alpha)^{\mathrm{lr}}+(\mu \mu \mid \beta \beta)^{\mathrm{lr}}+(\nu \nu \mid \alpha \alpha)^{\mathrm{lr}}+(\nu \nu \mid \beta \beta)^{\mathrm{l} r}\right] \\
& =\frac{1}{4} S_{\mu \nu} S_{\alpha \beta}\left[\gamma_{\mu \alpha}^{\mathrm{lr}}+\gamma_{\mu \beta}^{\mathrm{lr}}+\gamma_{\nu \alpha}^{\mathrm{lr}}+\gamma_{\nu \beta}^{\mathrm{lr}}\right]
\end{aligned}
$$




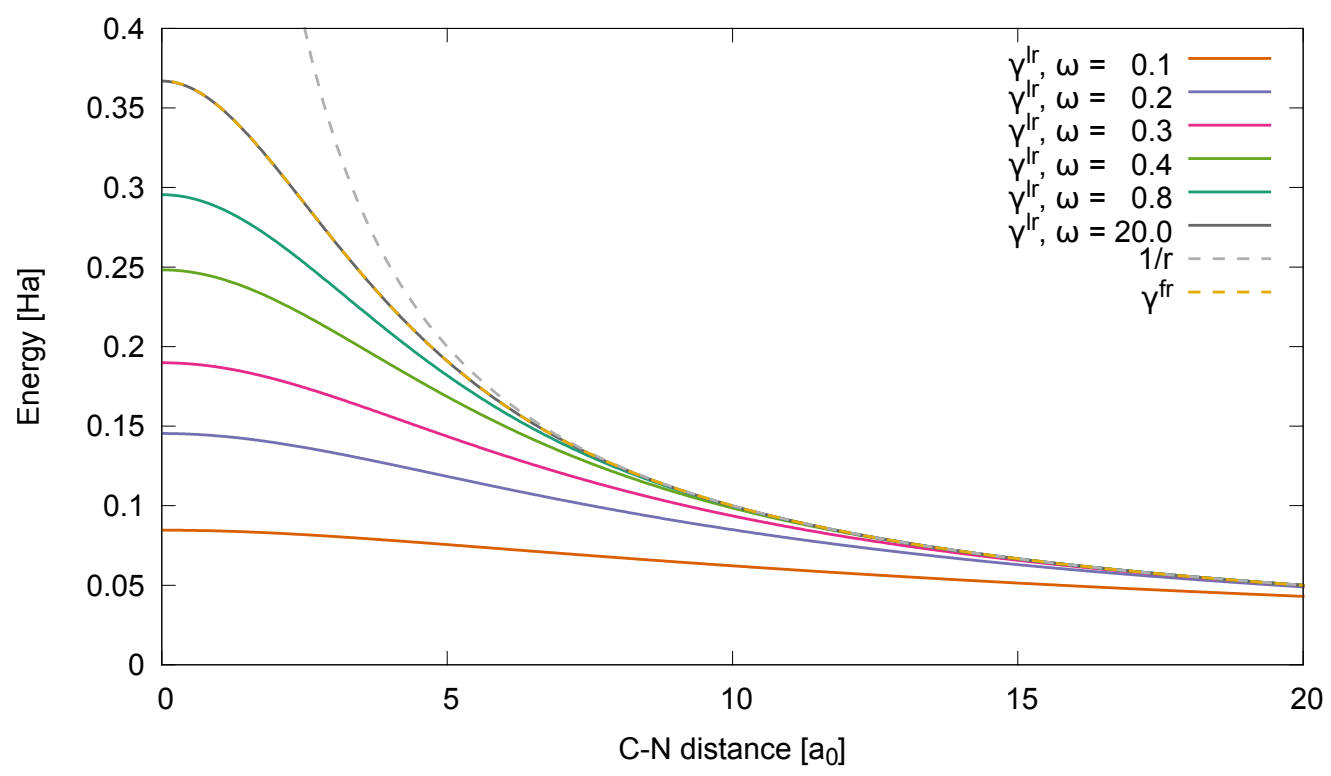

Figure 1: The long-range integral $\gamma^{\mathrm{lr}}$ for the carbon-nitrogen interaction as a function of inter-atomic distance for different values of the range-separation parameter $\omega$. The gray dashed line denotes the $1 / r$ limit. The full-range $\gamma^{\mathrm{fr}}$ as defined in appendix of Ref. 33 is depicted with an orange dashed line.

In the spirit of the original DFTB method, $\frac{33}{,}$ these integrals are then parametrized as

$$
\gamma_{\alpha \beta}^{\mathrm{lr}}=\gamma_{A B}^{\mathrm{lr}}\left(R_{A B}\right)=\frac{\tau_{A}^{3} \tau_{B}^{3}}{(8 \pi)^{2}} \iint e^{-\tau_{A}\left|\mathbf{r}_{1}-\mathbf{R}_{A}\right|} \frac{1-e^{-\omega\left|\mathbf{r}_{1}-\mathbf{r}_{2}\right|}}{\left|\mathbf{r}_{1}-\mathbf{r}_{2}\right|} e^{-\tau_{B}\left|\mathbf{r}_{2}-\mathbf{R}_{B}\right|} d \mathbf{r}_{1} d \mathbf{r}_{2},
$$

where $\alpha \in A, \beta \in B$, and $\tau_{A}$ is some atom specific decay constant, which has to be defined. In a similar fashion the full range two-electron integrals are simplified to

$$
\gamma_{\alpha \beta}^{\mathrm{fr}}=\gamma_{A B}^{\mathrm{fr}}\left(R_{A B}\right)=\frac{\tau_{A}^{3} \tau_{B}^{3}}{(8 \pi)^{2}} \iint e^{-\tau_{A}\left|\mathbf{r}_{1}-\mathbf{R}_{A}\right|}\left[\frac{1}{\left|\mathbf{r}_{1}-\mathbf{r}_{2}\right|}+f^{\omega, \times \mathrm{c}}\left[\rho_{0}\right]\left(\mathbf{r}_{1}, \mathbf{r}_{2}\right)\right] e^{-\tau_{B}\left|\mathbf{r}_{2}-\mathbf{R}_{B}\right|} d \mathbf{r}_{1} d \mathbf{r}_{2} .
$$

To obtain the final prescription for the evaluation of these integrals we follow the reasoning of the original DFTB method. For the off-site elements $A \neq B$, the contribution due to the xc-kernel $f^{\omega, x c}$ is assumed to vanish. Thus the full-range $\gamma$-integral reduces to a two-center Coulomb integral over simple spherically symmetric Slater type functions. For this case an analytical result is available, $\underline{33}$ which is also used in the present scheme. Exchange-correlation contributions are fully taken into account for the on-site case $A=B$, as shown below. To evaluate the long-range $\gamma$-integrals, we extend the analytical formula of Elstner et al ${ }^{[33}$ to the case of Yukawa interaction (see appendix $B$ for details). As example, we plot $\gamma_{\mathrm{C}-\mathrm{N}}^{\mathrm{lr}}$ for the carbon-nitrogen interaction as a function of inter-atomic 
distance for some values of the range-separation parameter $\omega$ in Figure 1. Additionally, the full-range $\gamma^{\text {fr }}$ evaluated with the analytical formula from Ref. 33 is also depicted (orange dashed line).

For the on-site elements $A=B$, the integrals have the form (details are given in appendix $B$ )

$$
\begin{aligned}
\lim _{R_{A B} \rightarrow 0} \gamma_{A B}^{\mathrm{lr}}\left(R_{A B}\right) & =\frac{5}{16} \tau_{A}-\frac{\tau_{A}^{8}}{\left(\tau_{A}^{2}-\omega^{2}\right)^{4}} \\
& \times\left[\frac{5 \tau_{A}^{6}+15 \tau_{A}^{4} \omega^{2}-5 \tau_{A}^{2} \omega^{4}+\omega^{6}}{16 \tau_{A}^{5}}-\omega\right] \\
\lim _{R_{A B} \rightarrow 0} \gamma_{A B}^{\mathrm{fr}}\left(R_{A B}\right) & =\frac{5}{16} \tau_{A} .
\end{aligned}
$$

For each element, the Hubbard parameter $\left(U=\partial^{2} E / \partial n^{2}\right)$ is defined as the second derivative of the energy with respect to the occupation of the highest occupied atomic orbital. The decay constants $\tau_{A}$ may therefore be determined by the requirement that LC-DFT and LC-DFTB yield the same Hubbard value for each species. In the presence of a Fock term this relation is different from that in traditional DFTB $\left(U_{A}=5 / 16 \tau_{A}\right)$. The Hubbard parameter of an atom in LC-DFTB is instead given by

$$
\begin{aligned}
U_{A}^{\mathrm{LC}-\mathrm{DFTB}} & =\gamma_{A A}^{\mathrm{fr}}-\frac{1}{2(2 l+1)} \gamma_{A A}^{\mathrm{lr}}=\frac{5}{16} \tau_{A}\left[1-\frac{1}{2(2 l+1)}\right. \\
& \left.\times\left(1-\frac{\tau_{A}^{8}+3 \tau_{A}^{6} \omega^{2}-\tau_{A}^{4} \omega^{4}+0.2 \omega^{6} \tau_{A}^{2}-3.2 \tau_{A}^{7} \omega}{\left(\tau_{A}^{2}-\omega^{2}\right)^{4}}\right)\right],
\end{aligned}
$$

where $l$ is the angular momentum of the highest atomic orbital (see appendix $\mathrm{C}$ for details). Evaluating $U_{A}$ using first principles LC-DFT for a given range-separation parameter $\omega$, Eq. 25 is solved numerically for $\tau_{A}$.

Having defined all elements of the method we proceed by applying the variational principle to the total energy in Eq. 15. This yields the Hamiltonian

$$
\begin{aligned}
H_{\mu \nu} & =H_{\mu \nu}^{0}+\frac{1}{4} \sum_{\alpha \beta} \Delta P_{\alpha \beta} S_{\mu \nu} S_{\alpha \beta}\left(\gamma_{\mu \alpha}^{\mathrm{fr}}+\gamma_{\mu \beta}^{\mathrm{fr}}+\gamma_{\nu \alpha}^{\mathrm{fr}}+\gamma_{\nu \beta}^{\mathrm{fr}}\right) \\
& -\frac{1}{8} \sum_{\alpha \beta} \Delta P_{\alpha \beta} S_{\mu \alpha} S_{\beta \nu}\left(\gamma_{\mu \beta}^{\mathrm{lr}}+\gamma_{\mu \nu}^{\mathrm{lr}}+\gamma_{\alpha \beta}^{\mathrm{lr}}+\gamma_{\alpha \nu}^{\mathrm{lr}}\right),
\end{aligned}
$$

and the generalized Kohn-Sham equations

$$
\sum_{\nu} H_{\mu \nu} c_{\nu, i}=\epsilon_{i} \sum_{\nu} S_{\mu \nu} c_{\nu, i}
$$

In contrast to the original DFTB scheme, the full density matrix needs to be evaluated self-consistently rather than just the Mulliken charges. 


\subsection{Implementation notes}

The scheme has been implemented in the development version of the DFTB + code. ${ }^{36}$ With respect to the original DFTB method, two significant changes are necessary. First, the zeroth-order Hamiltonian (Eq. 17) now also includes a contribution due to screened Hartree-Fock exchange. The necessary adaption of the numerical integration routines is outlined in appendix A. Since the $H^{0}$ and $S$ matrix elements are precomputed there is no overhead involved in actual calculations.

The second change is related to the presence of an exchange term in the Fock matrix, given by the second line in Eq. 26. This part is performance-critical, since a naive implementation would lead to $N^{4}$ scaling, despite of the applied Mulliken approximation (Eq. 18).

We therefore resort to techniques which are widely used in direct SCF calculations. ${ }^{[35]}$ The Hamiltonian is constructed iteratively

$$
H\left(\mathbf{P}^{n}\right)=H\left(\mathbf{P}^{n-1}+\Delta \mathbf{P}\right)=H\left(\mathbf{P}^{n-1}\right)+H(\Delta \mathbf{P}),
$$

such that the Hamiltonian at the $n$-th iteration is the sum of the Hamiltonian at the previous iteration plus a correction. Here the density matrix at $n$-th iteration is labeled $\mathbf{P}^{n}$ and $\Delta \mathbf{P}$ denotes the difference between density matrices at different iterations of the self-consistent cycle, not the difference between $\mathbf{P}$ and $\mathbf{P}_{\mathbf{0}}$ as in Eq. 14.

We then exploit the fact that the matrix elements $H(\Delta \mathbf{P})$ get smaller upon approaching convergence. In order to make efficient use of integral prescreening techniques, the exchange matrix in Eq. 26 is rewritten in the following form

$$
\begin{aligned}
K_{\mu \nu} & =-\frac{1}{8} \sum_{\alpha \beta} \Delta P_{\alpha \beta}^{n} S_{\mu \alpha} S_{\beta \nu}\left(\gamma_{\mu \beta}^{\mathrm{lr}}+\gamma_{\mu \nu}^{\mathrm{lr}}+\gamma_{\alpha \beta}^{\mathrm{lr}}+\gamma_{\alpha \nu}^{\mathrm{lr}}\right) \\
& =-\frac{1}{8} \sum_{A B} \underbrace{\left[\gamma_{C B}^{\mathrm{lr}}+\gamma_{A B}^{\mathrm{lr}}+\gamma_{C D}^{\mathrm{lr}}+\gamma_{A D}^{\mathrm{lr}}\right]}_{\Gamma_{A B C D}} \sum_{\alpha \in A} \sum_{\beta \in B} S_{\mu \alpha} \Delta P_{\alpha \beta}^{n} S_{\beta \nu} \\
& =-\frac{1}{8} \sum_{A B} \Gamma_{A B C D} Q_{A B}^{\mu \nu} .
\end{aligned}
$$

For each Hamiltonian sub-block (C,D) and atom pair (A,B), an estimate for the quantities $Q_{A B}^{\mu \nu}, \mu \in$ $C, \nu \in D$ is given by

$$
Q_{A B}^{\mu \nu} \leq \sum_{\alpha \in A} \sum_{\beta \in B}\left|S_{\mu \alpha}\right|\left|\Delta P_{\alpha \beta}^{n}\right|\left|S_{\beta \nu}\right| \leq \bar{S}_{B D} \bar{S}_{A C} \overline{\Delta P^{n}}\left(\sum_{\alpha \in A} \sum_{\beta \in B} 1\right),
$$

where $\bar{S}_{A B}=\max _{\alpha \in A, \beta \in B}\left(\left|S_{\alpha \beta}\right|\right)$ and $\overline{\Delta P^{n}}=\max \left(\left|\Delta P_{\alpha \beta}^{n}\right|\right)$. Imposing the cutoff criterion

$$
\bar{S}_{B D} \bar{S}_{A C} \overline{\Delta P^{n}} \leq \epsilon_{\text {threshold }}
$$




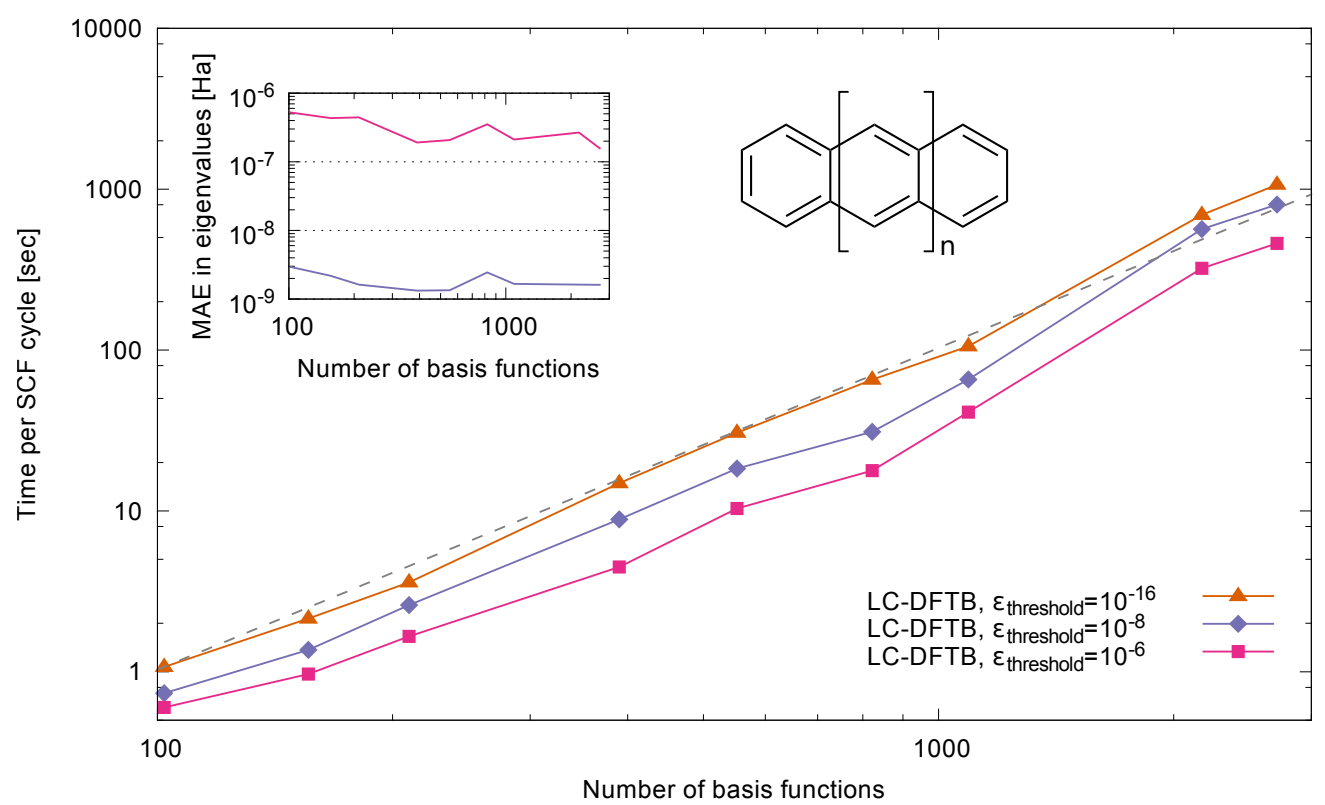

Figure 2: The average time per SCF cycle for the polyacene oligomers versus the number of basis functions for the threshold parameters $\epsilon_{\text {threshold }}=10^{-16}, 10^{-8}, 10^{-6}$. The gray dashed line gives an extrapolation for ideal quadratic scaling. The inset shows the mean absolute error in the eigenvalues in Hartree for the cases of $\epsilon_{\text {threshold }}=10^{-8}$ and $\epsilon_{\text {threshold }}=10^{-6}$. Calculations have been performed on a single core of an Intel Core-i7 CPU. The execution time was measured by the Linux time utility.

we decide whether the contribution of the Hamiltonian matrix element is significant and avoid the evaluation if possible. The double sum on the right side of Eq. 32 is absorbed in $\epsilon_{\text {threshold }}$.

We analyze the efficiency and accuracy of this prescreening approach by performing benchmark calculations for the polyacene series $\left(C_{4 n+2} H_{2 n+4}\right)$ up to $n=150$. Figure 2 depicts the scaling of the method with respect to the basis size. The gray dashed line shows the extrapolated quadratic function $t(n)=c n^{2}$, with $t\left(n_{0}\right)$ equal to the CPU time at the smallest oligomer size $n_{0}=5$ and $\epsilon_{\text {threshold }}=10^{-16}$.

For $\epsilon_{\text {threshold }}=10^{-16}$, prescreening and exact evaluation according to Eq. 29 lead to identical total energies within machine precision. Even with this tight threshold criterion, the expected quadratic scaling is achieved. For the finite threshold values of $\epsilon_{\text {threshold }}=10^{-8}$ and $\epsilon_{\text {threshold }}=10^{-6}$ the scaling remains quadratic, however the prefactor is reduced by a factor of 2-3. In the inset the mean absolute error (MAE) of the eigenvalues with respect to the exact evaluation is shown. For $\epsilon_{\text {threshold }}=10^{-6}$ this error does not exceed $10^{-6} \mathrm{Ha}$.

The overall scaling of the method is determined by the calculation step with steepest scaling. Since the Hamiltonian matrix construction scales quadratically in our scheme, the scaling for very large 
systems should be determined by the $\mathcal{O}\left(N^{3}\right)$ behavior of the diagonalizer. However, for the tested systems the time spent for diagonalization is negligible compared to the Hamiltonian construction. Thus we expect cubic scaling only for much larger system sizes, especially if effective Divide \& Conquer diagonalization routines are available like in the current DFTB+ version.

\section{RESULTS}

After the discussion of the main approximations and computational efficiency of the LC-DFTB method, we benchmark its predictive power. At this point we focus on the electronic structure at fixed geometry to highlight the advantages with respect to the original DFTB method in the computation of electron removal and addition energies. This also represents a necessary first step in developing a time-dependent formalism for long-range corrected functionals in the spirit of the TD-DFTB scheme.

\subsection{Quasi-particle energies}

It is well known that KS eigenvalues from local DFT are poor estimates for quasi-particle energies.22] 37-41 Especially the eigenvalue of the highest occupied molecular orbital (HOMO), if interpreted as electron removal energy (ionization potential) underestimates the experimental ionization potential (IP) by several eV. Electron affinities estimated from energy of the lowest unoccupied molecular orbital (LUMO) are likewise prone to large errors. As a consequence the HOMO-LUMO gap is much smaller than the experimental fundamental gap or the one obtained from the GW approximation. 38 42-44 This challenges the ability of the local DFT to provide useful single-particle picture of the physical systems.

Two major problems of local DFT have been identified. On the one hand, the exponential asymptotic decay of the KS-potential (instead of the correct $-1 / r$ behavior) leads to underbound electrons and a wrong description of the long-range interaction. On the other hand, the correct asymptotics alone is usually not sufficient for the correct prediction of quasi-particle energies. ${ }^{38}$ The correct total energy of a system with fractional occupation $N+\delta$, where $N$ is an integer and $0 \leq \delta \leq 1$ is real, has been shown to exhibit a linear dependence on particle number between $N$ and $N+1$. This is the necessary and sufficient condition to obtain the correct IP and fundamental gap. The linearity condition has been directly connected to the many-electron self-interaction error (MSIE) exhibited by local exchange-correlation functionals, $\frac{45}{4}$ which in a real space picture manifests as a delocalization problem. $\underline{38}$

The LC-DFT restores the correct asymptotics of the potential and shows remarkably close agreement with the linearity condition. $22[38|39| 46$ As a consequence, at least frontier orbitals in LC-DFT 
can be interpreted as electron removal energies. We expect the LC-DFTB method to show similar qualitative improvement compared to DFTB.

To investigate this point, we choose a set of organic molecules for which experimental ion energetics data is available. Among others this set includes a selection of compounds that are relevant for photovoltaic applications and have been studied in a similar context with LC-DFT ${ }^{44}$ and GW 42 methods. The structural formulas of these molecules can be found in the supporting material.

All geometries have been optimized at the traditional DFTB level with the mio-1-1 set ${ }^{33}[34$ and are used by default for both first principles and DFTB/LC-DFTB calculations. For the calculations in this work, we choose a value of $\omega=0.3 \mathrm{a}_{0}^{-1}$ for the range-separation parameter unless stated otherwise. We found this value to give reasonable results for the prediction of ionization potentials. Akinaga and Ten-no showed, that for a range-separation of Yukawa type the optimal value of the range-separation parameter is usually higher than that for error function based functionals. ${ }^{28}$ In their work the optimization of the parameter was carried out on atomization energies of the G2-1 set with the cc-pVTZ basis. However, it is so far not clear whether these parameters can be directly transferred to LC-DFTB. From our calculations, we found values ranging from $\omega=0.5 \mathrm{a}_{0}^{-1}$ to $\omega=0.75 \mathrm{a}_{0}^{-1}$, as suggested in Ref. 28, to be too large for the prediction of accurate ionization potentials. In this case LC-DFTB tends to systematically overestimate IPs.

We include the gradient-corrected PBE ${ }^{29}$ functional, the hybrid functional B3LYP $47-50$ and the long-range corrected $B{ }^{25}$ functional in the comparison. In general a basis set of triple-zeta quality with polarization functions is employed, while we also use smaller sets for the BNL functional to estimate the basis set dependence of the results. All first principles DFT calculations have been performed with the NWCHEM-6.3 package. 51

First we compare the negative of the HOMO eigenvalue to the experimental ionization potentia 52 for the mentioned set of molecules. The deviation $\Delta=\left|I P_{\text {exp }}\right|-\left|\epsilon_{\text {HOMO }}\right|$ for LC-DFTB, DFTB, PBE, B3LYP and BNL is shown in the top part of Figure 3. It is found that LC-DFTB is quantitatively in better agreement with experiment than the standard DFTB and first principle approaches with the PBE and B3LYP functionals. The MAE for LC-DFTB is $0.50 \mathrm{eV}$ compared to BNL/3-21G (0.67 $\mathrm{eV}), \mathrm{BNL} / \mathrm{cc}-\mathrm{pVDZ}(0.47 \mathrm{eV})$, and BNL/cc-pVTZ (0.29 eV). Since the deviations for the local and hybrid functionals are much larger [B3LYP/cc-pVTZ (2.04 eV), PBE/cc-pVTZ (2.87 eV), DFTB $(2.50 \mathrm{eV})]$, we conclude that the assets of long-range corrected functionals are still visible at the approximated LC-DFTB level.

For some molecules, most notably thiadiazole and methane, the deviation from both experiment and BNL can however be quite large. This seems to be an effect of the basis set employed in the 


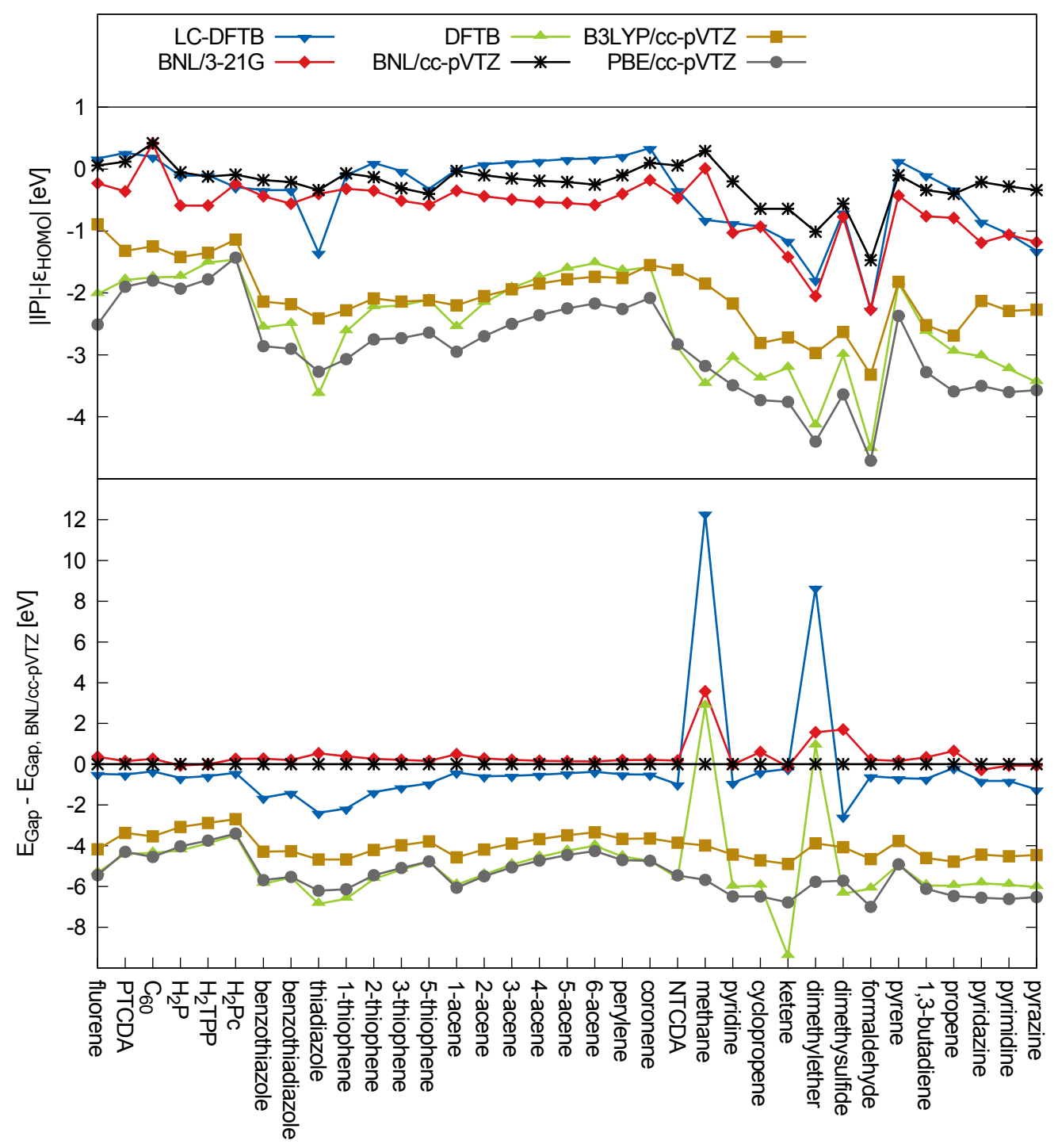

Figure 3: (Top) Deviation of the negative HOMO eigenvalue $\left(-\epsilon_{\mathrm{HO} о \mathrm{O}}\right)$ from the experimental ionization potential for different levels of theory. (Bottom) Deviation of the HOMO-LUMO gap from the reference (BNL/cc-pVTZ). 
LC-DFTB method. As already mentioned in Section 2, we did not optimize the compression radii for the new functional and use the parameters of the mio-1-1 basis set without changes. Furthermore the minimal basis of the DFTB/LC-DFTB method might not provide enough variational flexibility. This can be deduced from the results for the BNL functional at the 3-21G, cc-pVDZ and cc-pVTZ level. Thiadiazole is an exception, since for this compound basis set effects in the first principles calculations (BNL/3-21G compared to BNL/cc-pVTZ) are small. Inspection of Figure 3 reveals that the results of the LC-DFTB method are in general comparable to LC-DFT results with small doublezeta basis (3-21G). The new scheme clearly outperforms first principles DFT calculations based on the PBE and B3LYP functionals for the description of ionization potentials.

Next, we investigate fundamental band gaps. Since the experimental data for electron affinities is in general not available, we use BNL/cc-pVTZ results as reference. Deviations of the fundamental gaps from this reference are depicted in the bottom part of Figure 3. The MAE deviation for LC-DFTB is $1.36 \mathrm{eV}$, compared to DFTB (5.06 eV), BNL/3-21G (0.41 eV), BNL/cc-pVDZ (0.15 eV), PBE/ccpVTZ $(5.29 \mathrm{eV})$, and B3LYP/cc-pVTZ $(3.92 \mathrm{eV})$. For the case of methane and dimethylether rather large deviations of $12.30 \mathrm{eV}$ and $8.66 \mathrm{eV}$, respectively, are found for LC-DFTB. Again we assign these failures to the minimal basis set, since $\mathrm{BNL} / 3-21 \mathrm{G}$ shows qualitatively similar, although much smaller, deviations of $3.58 \mathrm{eV}$ and $1.57 \mathrm{eV}$ for these molecules. In line with this we note that dimethylsulfide, which is essentially dimethylether with oxygen being replaced by sulfur, shows a much smaller error. This can be attributed to the fact that the sulfur in present parametrization contains additional polarization functions (d-orbitals). Inclusion of polarization functions for oxygen and nitrogen might thus reduce the mentioned problem with a moderate loss of computational efficiency.

We now proceed by investigating the electronic structure beyond the frontier molecular orbitals which is experimentally accessible by photoemission spectroscopy. Here successful applications of theoretical methods such as GW or hybrid DFT have been reported, whereas local DFT exhibited serious flaws. $.33[53-55$ The deficiencies of local DFT, which could be attributed to the self-interaction error, seem to be partially cured by long-range corrected functionals. ${ }^{[37}[46][53]$ The eigenvalue spectrum from standard DFTB, LC-DFTB, PBE/cc-pVTZ and BNL/cc-pVTZ theories for 3,4,9,10-perylenetetracarboxylic-dianhydride (PTCDA) and pentacene (5-acene) molecules is presented in Figure 4. All spectra are rigidly shifted such that $\mathrm{HOMO}$ position is at $0 \mathrm{eV}$. We use a simple gaussian broadening profile with the full width at half minimum of $0.1 \mathrm{eV}$ to mimic the experimental resolution and broadening. For this study geometries optimized at the B3LYP/cc-pVTZ level have been used.

The experimental photoemission spectrum of the PTCDA molecule is characterized by the large gap of $1.5 \mathrm{eV}$ between the first and second peaks, where the second peak appears at energies relative to the $\mathrm{HOMO}$ between $-1.5 \mathrm{eV}$ and $-2.1 \mathrm{eV} \cdot{ }^{56}$ Long-range corrected functionals and $\mathrm{GW}$ are usually 


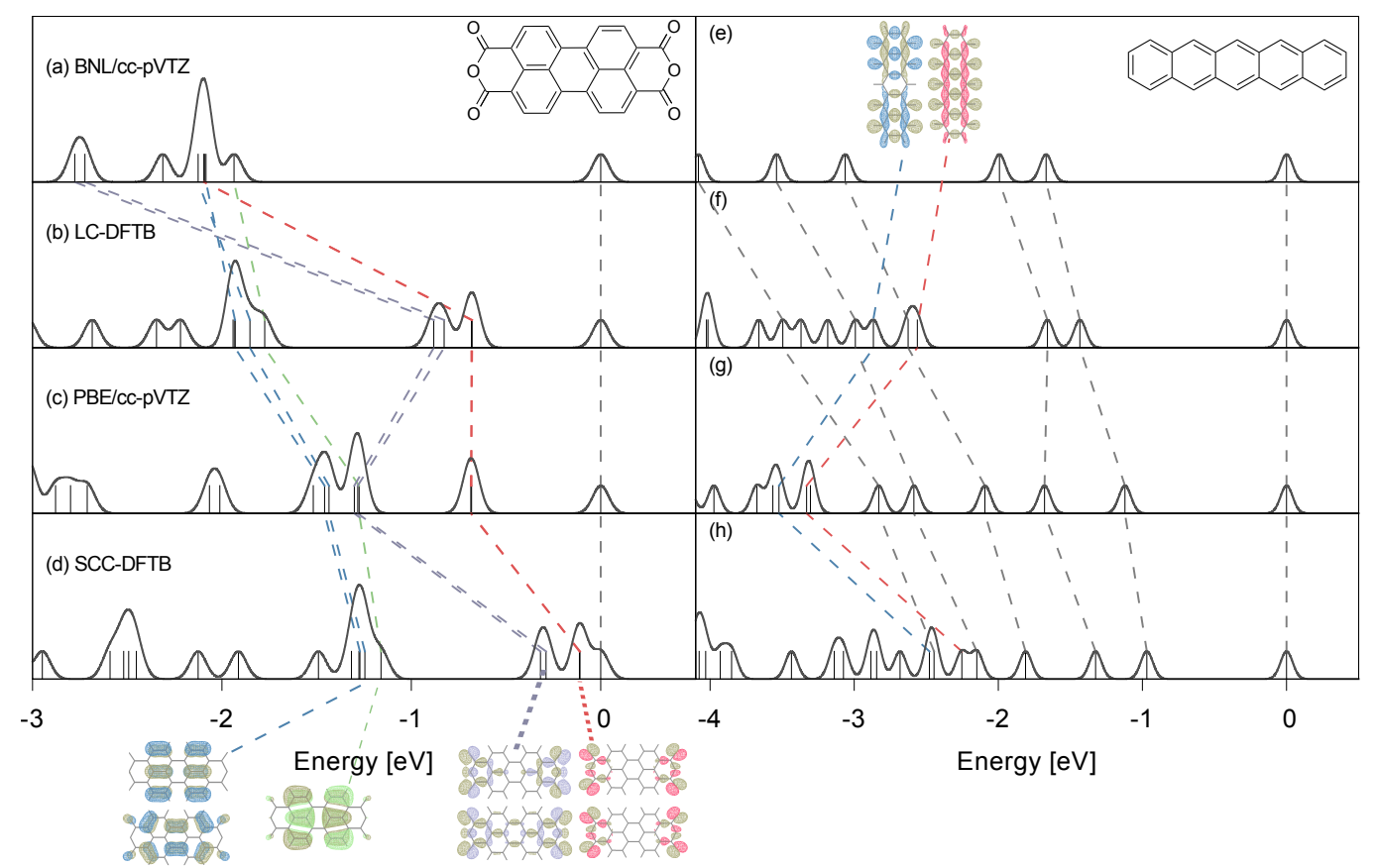

Figure 4: Broadened density of states for PTCDA and pentacene molecules from LC-DFTB and DFTB compared to BNL/cc-pVTZ and PBE/cc-pVTZ results. The HOMO level has been shifted to the zero of energy for all methods.

capable to reproduce these features qualitatively. ${ }^{23}$ We confirm this again with our BNL/cc-pVTZ calculation, see part a) of Figure 4 . The spectrum is comparable to the standard LC- $\omega$ PBE result of Ref. 46. Since PBE provides poor results as has been already discussed in Refs. 37, 46, we do not expect a better performance for DFTB, which is essentially an approximate DFT with the PBE functional. As can be clearly seen from part c) and d) of Figure 4, the DFTB spectrum differs even more strongly from BNL/cc-pVTZ than PBE.

We keep this fact in mind and proceed to the LC-DFTB results. We observe again a major difference to the BNL/cc-pVTZ spectrum. The LC-DFTB shows four $\sigma$-orbitals, mostly located at the anhydride groups of the PTCDA, right in the middle of the mentioned gap. These orbitals fit into the level ordering scheme of DFTB, therefore it seems that LC-DFTB just shifts the levels of DFTB if the range-dependent HF exchange term is added. However, we find for other systems that the shift is non-uniform and the level ordering is not preserved (see below the discussion of pentacene). For long-range corrected functionals the transition from $\omega=0$ (DFT limit) to finite $\omega$ values is quite generally accompanied by smooth inhomogenous shifts of the single particle levels. Therefore level reordering is expected. Obviously the BNL/cc-pVTZ theory correctly exhibits this reordering of levels with respect to PBE/cc-pVTZ, which is not observed for LC-DFTB in the example shown here. This should be attributed to the typical DFTB approximations, like the reduced basis set and the 
two-center approximation, since already the DFTB results in Figure 4d) show strongly underbound $\sigma$-orbitals. As level ordering issues have also been observed in first principles LC-DFT, there can also be an additional effect. The analysis in Ref. 46 showed, that even if the frontier orbitals seem to be well described by LC-DFT, there are states, usually of different symmetry (e.g. $\sigma$-orbitals), which exhibit considerable orbital SIE (OMSIE). In the aforementioned work the spectrum of tuned LC$\omega$ PBE functional has been discussed. Within the tuning procedure the value of the range-separation parameter is chosen such that the HOMO eigenvalue is equal to the ionization energy obtained from the difference of total energies of the neutral species and the cation. For this functional the second peak in the PTCDA spectrum is composed of degenerate $\sigma$ states (which correspond to the HOMO2/HOMO-3 in the LC-DFTB spectrum in the present work). Analysis of the orbital self-interaction error for this theory showed large OMSIE in the $\sigma$-orbitals, while for $\pi$-orbitals the error was small. LC- $\omega$ PBE with standard value of the range-separation parameter exhibited the opposite behavior, where the $\sigma$-orbitals had rather small OMSIE. Thus the failure of LC-DFTB could also be connected to residual self-interaction error which is more pronounced for the anhydride $\sigma$-orbitals. In fact, the energetical position of the $\pi$ orbitals relative to the HOMO level is quite well represented by LC-DFTB, much better than in first principles PBE.

The problematic level ordering of LC-DFTB is also observed for the pentacene spectrum (right part of Figure 4). While the levels up to HOMO-4 in DFTB, PBE and BNL show the same order, the LC-DFTB spectrum is characterized by the appearance of two $\sigma$-orbitals at HOMO- 3 and HOMO-5 positions. They are indicated by red and blue lines respectively. It turns out that these orbitals have already a differing position in the DFTB spectrum as compared to PBE. The approximate theories tend to underbind these orbitals. Thus in this case the influence of the DFTB approximations is more evident. From the analysis above, we conclude that the level ordering problem of the LC-DFTB might be partially caused by the orbital self-interaction error of the $\sigma$-states within the LC-DFTB theory as well as by the applied DFTB approximations and the minimal basis set used. Again we find a significant spreading of the quasi-particle spectrum in line with the LC-DFT results and quite accurate level positions for the HOMO, HOMO-1 and HOMO-2 levels of $\pi$-nature. Notwithstanding, a full characterization of an experimental photoemission spectrum seems to be too ambitious at this point.

At the end of this section we briefly comment on the computational efficiency of the new scheme versus first principles approaches. In fact, the main motivation to use approximate methods like LCDFTB is the possibility to investigate large systems well beyond the scope of conventional DFT codes. In Section 2.3 we already documented the quadratic scaling of the method with system size. In Table 2 absolute timings of single point calculations for the theories considered in the previous discussion 


\begin{tabular}{lrrrrrr}
\hline \hline molecule & BNL/3-21G & BNL/cc-pVDZ & BNL/cc-pVTZ & LC-DFTB $^{1}$ & LC-DFTB $^{2}$ & DFTB $^{-}$ \\
\hline 5-Acene & 325 & 2396 & 59701 & 11 & 6 & 1 \\
Perylene & 541 & 4447 & 113414 & 12 & 6 & 1 \\
$\mathrm{H}_{2} \mathrm{P}$ & 932 & 7486 & $23825^{*}$ & 16 & 8 & 1 \\
Coronene & 2116 & 15983 & 260677 & 14 & 6 & 1 \\
6-Acene & 507 & 3516 & 79993 & 15 & 8 & 2 \\
5-Thiophene & 1303 & 11581 & 144735 & 16 & 11 & 2 \\
PTCDA & 3748 & 20477 & 521424 & 22 & 10 & 2 \\
$\mathrm{H}_{2}$ Pc & 3034 & 27231 & $366838^{*}$ & 39 & 14 & 2 \\
$\mathrm{H}_{2}$ TPP & 5735 & 43304 & $744967^{*}$ & 72 & 22 & 4 \\
$\mathrm{C}_{60}$ & 7221 & 65393 & $655789^{*}$ & 121 & 23 & 2 \\
\hline \hline
\end{tabular}

Table 2: Wall time [s] of DFT calculation versus LC-DFTB for molecules with more than 30 atoms from the considered set. The DFTB results are given for comparison as well. The asterisk denotes the parallel jobs on 12 CPUs, LC-DFTB ${ }^{1}$ was performed with $\epsilon_{\text {threshold }}=10^{-16}$ and LC-DFTB $^{2}$ with $\epsilon_{\text {threshold }}=10^{-6}$.

are summarized. The LC-DFTB calculations, with $\epsilon_{\text {threshold }}=10^{-16}$ (denoted by LC-DFTB $^{1}$ ) and $\epsilon_{\text {threshold }}=10^{-6}$ (denoted by LC-DFTB ${ }^{2}$ ) have been performed on a single core of an Intel Core-i7 $\mathrm{CPU}$ as before. The execution time was measured by the Linux time utility, where the user time in seconds has been collected. For the DFT calculations the NWCHEM- 6.3 code was used in serial and parallel versions. The serial version of NWCHEM-6.3 was executed on Intel Xeon $2.8 \mathrm{GHz}$ machines, while the parallel jobs were distributed over $12 \mathrm{CPUs}$ on a cluster. Wall times were extracted from the NWCHEM- 6.3 output files.

As expected, the first-principles calculations are computationally more demanding. Even the calculation with small basis set at the BNL/3-21G theory level is at least 30 times slower than LCDFTB $^{1}$ and 50 times slower than LC-DFTB ${ }^{2}$ for smaller molecules. We note that the threshold parameter $\epsilon_{\text {threshold }}=10^{-6}$ gives the eigenvalues with MAE errors below $10^{-5} \mathrm{eV}$ (see Figure 2), thus this choice can be considered as accurate for practical calculations. For larger systems the gap in computational time between the LC-DFTB and first-principles calculations increases due to the quadratic scaling of the LC-DFTB method. At the same time traditional DFTB is at least an order of magnitude faster than LC-DFTB with $\epsilon_{\text {threshold }}=10^{-6}$. 


\subsection{Electric field response: DFTB vs. LC-DFTB}

A well known deficiency of local DFT is the exaggerated response to an applied electrostatic field. This behavior have been attributed to the lack of a necessary non-local response term in the exchangecorrelation functional.57-59 Thus all local and semilocal exchange-correlation functionals fail to produce the correct induced field, which is opposed to the applied electric field. As a consequence, local DFT leads to a wrong density distribution, characterized by a too strong separation of the induced charge. This results in a drastic overestimation of static polarizabilities, which gets stronger with growing system size. Difficulties amplify for the hyperpolarizability and second hyperpolarizability ${ }^{60}$ This problem has also consequences for the application of DFT in the field of molecular electronics. The underestimated HOMO-LUMO gap, lack of a field-counteracting term and delocalization of the density lead altogether to a flawed description of transport properties, such as conductance. ${ }^{61} \mathrm{We}$ showed already in the section 3.1, that the LC-DFTB provides essentially better description of the fundamental gap, which suggests the reduction of the delocalization problem as compared to traditional DFTB. Recently, Sekino et al. provided evidence that LC-DFT shows the tendency to overcome the field response problem. ${ }^{62}$ We therefore seek to confirm the signatures of a field-counteracting term due to the inclusion of the non-local range-dependent term in the LC-DFTB method. We calculate the static longitudinal polarizabilities of polyacetylene chains $\left(P A, \mathrm{C}_{2 n} \mathrm{H}_{2 n+2}\right.$ ) with varying number of unit cells $n$ and analyze the induced Mulliken charge distribution along the chain. LC-DFTB as well as traditional DFTB include the electric field $\mathbf{F}$ via the additional contribution

$$
E_{\text {field }}=-\sum_{A} \Delta q_{A} \mathbf{F} \cdot \mathbf{R}_{A}
$$

to the total energy functional, where $\Delta q_{A}=q_{A}-q_{A}^{0}$ is the difference of the Mulliken population $q_{A}=\sum_{\nu \in A}(\mathbf{P S})_{\nu \nu}$ and the number of valence electrons on atom $A$ located at position $\mathbf{R}_{A}$. We point out that traditional DFTB (and for the same reasons LC-DFTB) shows in general poor performance in predicting polarizabilities. This is due to the minimal basis set employed. However, LC-DFT even with minimal basis (e.g. BNL/STO-3G) tends to correctly reduce the polarizability with respect to LDA/GGA-based theory in the same basis.

We compare the polarizabilities obtained from the LC-DFTB method to long-range corrected DFT at the BNL/6-311G** and BNL/3-21G level. For the case of LC-DFT the polarizabilities have been obtained from coupled-perturbed Kohn-Sham theory (CPKS), implemented in the NWCHEM-6.3 package. The geometries for PA with $n=10$ and $n=40$ have been optimized at the B3LYP/6311G* level of theory. We obtain the LC-DFTB polarizabilities by applying the finite field method. The numerical derivative of the dipole moment $\mu$ along the long axis with respect to the perturbing electric field $F$ is calculated with the center difference formula $\alpha=(\mu(F)-\mu(-F)) / 2 F$, where the 


\begin{tabular}{lccc}
\hline \hline$\omega\left[a_{0}^{-1}\right]$ & BNL/6-311G** & BNL/3-21G & LC-DFTB \\
\hline 2.0 & 1288 & 1229 & 1156 \\
0.8 & 1212 & 1138 & 1200 \\
0.5 & 1193 & 1102 & 1256 \\
0.3 & 1321 & 1215 & 1345 \\
0.2 & 1513 & 1400 & 1429 \\
0.1 & 1809 & 1696 & 1560 \\
$10^{-2}$ & 2058 & 1938 & 1698 \\
$10^{-3}$ & 2059 & 1939 & 1701 \\
\hline
\end{tabular}

Table 3: Static longitudinal polarizability of PA $(n=10)$. All values are in atomic units $\left[a_{0}^{2} e^{2} / E_{h}\right]$.

field strength was chosen to be $\mathrm{F}=0.0004$ a.u.. Table 3 lists the longitudinal static polarizability of the PA oligomer with $n=10$ for BNL/6-311G**, BNL/3-21G and LC-DFTB at different values of the range-separation parameter $\omega$. We observe similar qualitative behavior, although the quantitative differences are rather large, especially for the local DFT limit $(\omega \rightarrow 0)$. In this local DFT limit all three theories exhibit larger polarizabilities as in the opposite $\mathrm{HF}+\mathrm{c}$ limit $(\omega \rightarrow \infty)$. In the latter case the exchange-correlation functional is composed of $100 \% \mathrm{HF}$ exchange and local DFT correlation. Remarkable is the rapid drop of the polarizability with the increase of the range-separation parameter $\omega$ (compare also Figure 5), seen in all theories. While in the case of $n=10$ the ratio of $\mathrm{HF}+\mathrm{c}$ to local DFT limits is 0.63 for both BNL/6-311G** and BNL/3-21G, and 0.68 for LC-DFTB, it decreases to 0.23 for $\mathrm{BNL} / 3-21 \mathrm{G}$ and 0.33 in the case of LC-DFTB for the larger system with $n=40$ units. This indicates the aforementioned growing of the polarizability overestimation with the increasing system size.

Further information is obtained by inspection of the charge density. The inset of Figure 5 depicts the induced Mulliken charge due to a field of magnitude $\mathrm{F}=0.001 \mathrm{a}$.u. along the $n=40$ oligomer for different values of the range-separation parameter $\omega$. The LC-DFTB $(\omega \rightarrow 0)$ shows an almost linear charge distribution, which indicates an overly large polarization. We note that the DFTB is quantitatively very close to the LC-DFTB in the DFTB limit $\omega \rightarrow 0$, although the exchangecorrelation functional is slightly different. Thus we do not show the DFTB result for brevity. Increase of the parameter $\omega$ gives rise to an effective screening of the electric field, which leads to the correction of the polarizability towards more physical values. The LC-DFTB polarizability as a function of the range-separation parameter is provided in the main part of Figure 5 for comparison. We emphasize the qualitative nature of the LC-DFTB results presented here. Quantitatively correct polarizabilities require in general large basis sets. An alternative solution is the exploitation of empirical correction 


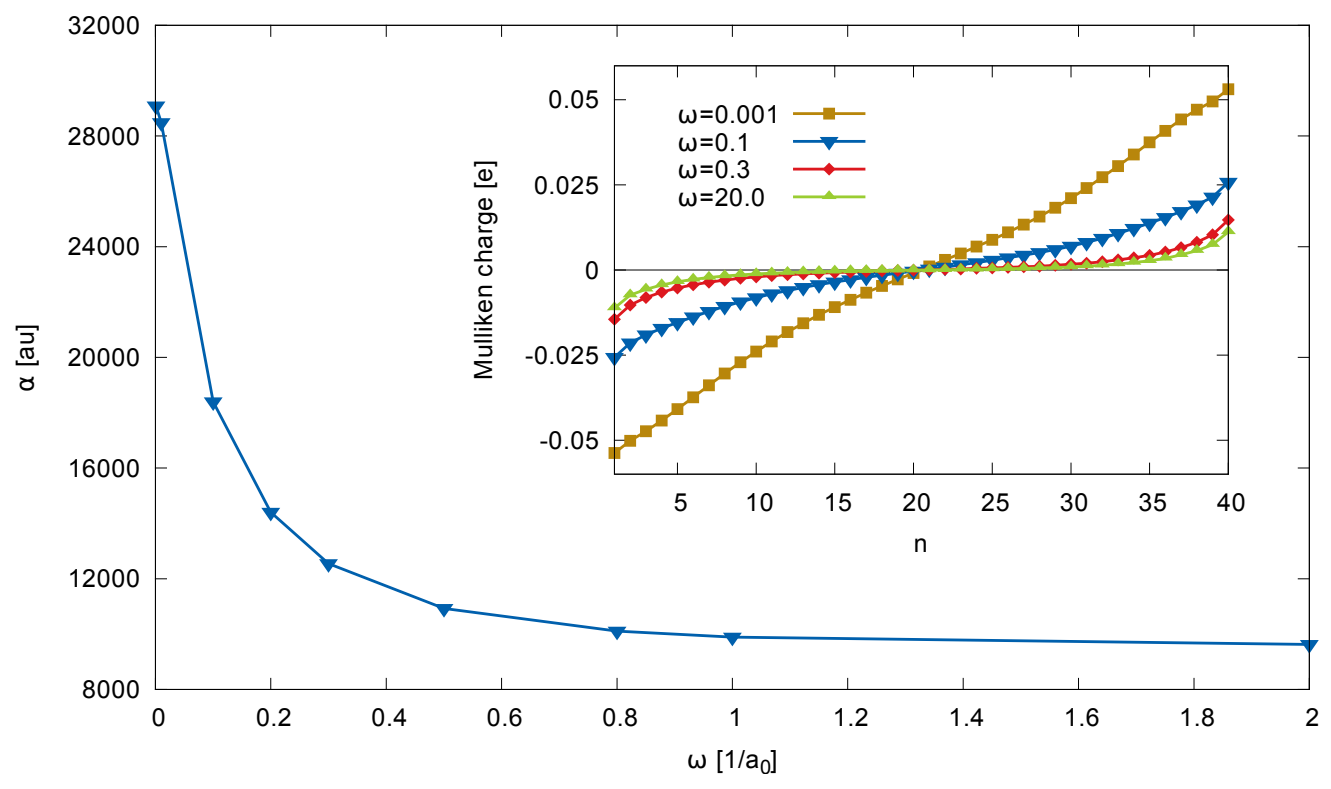

Figure 5: LC-DFTB linear polarizability of the PA $(n=40)$ oligomer as a function of range-separation parameter $\omega$. The induced Mulliken charge per unit $(F=0.001$ a.u. $)$ for different values of $\omega$ is shown in the inset.

methods $[6364$

\subsection{Proteins in gas phase and solution}

Experimental techniques that allow for the non-destructive extraction of proteins in combination with structural analysis make it possible to study intramolecular interactions in the absence of a particular solvent. ${ }^{65}$ Electrospray ionization $(E S I)^{66}$ together with mass spectrometry and ion mobility measurements permit gas-phase structure determination. Likewise, recent progress in the development of X-ray free-electron lasers hold the promise to resolve protein structure in vacuum at atomic resolution.67 68 From the theoretical side, computations using solvent models in addition to gas phase calculations may finally provide a way to understand the protein folding mechanism in different environments.

Efforts in this direction may address the still controversial question whether peptides adopt the zwitterionic form known from aqueous solution also in the gas phase. 69 DFTB, like other DFT approaches based on local xc-functionals, shows difficulties in the description of the zwitterionic state where long-range charge-charge interactions play an important role. In a recent study Nishimoto et al. ${ }^{72}$ found that the DFTB self-consistency cycle failed to converge for the model peptides chignolin (PDB ID: $1 \mathrm{UAO}^{73}$ ) and Trp-cage (PDB ID: $1 \mathrm{~L}^{2 Y^{74}}$ ) in the zwitterionic conformation. It has been, however, shown both theoretically with force-field based molecular dynamics and experimentally using 


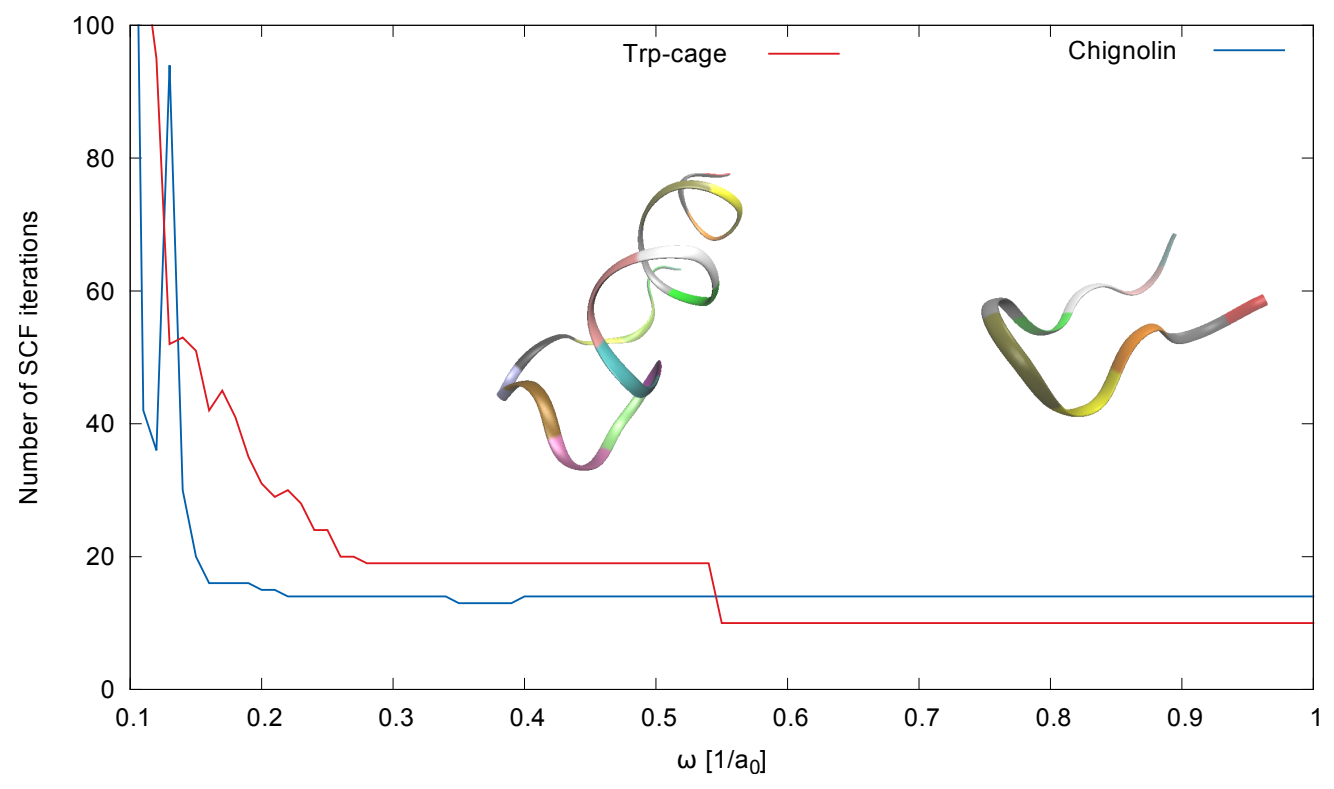

Figure 6: The SCF convergence plot of chignolin and Trp-cage proteins as a function of rangeseparation parameter $\omega$ for the LC-DFTB method. The SCF does not converge in the local DFTB limit $(\omega=0)$. Structures of the proteins are shown as insets. These plots have been generated from PDB structures ${ }^{73}\left[74\right.$ with the VMD software. ${ }^{77}$

electrospray ionization and photo-dissociation, that the native zwitterionic configuration of Trp-cage remains stable in the gas-phase ${ }^{75}, 76$

Raising the electronic temperature seems to circumvent the issue as a technical workaround, but in fact cures the symptoms instead of solving the fundamental problem, which is the notorious underestimation of the HOMO-LUMO gap of traditional DFTB. Motivated by this we present singlepoint calculations on the $1 \mathrm{UAO}$ and $1 \mathrm{~L} 2 \mathrm{Y}$ structures using the LC-DFTB method and compare to the LC-PBE/3-21G and RHF/3-21G methods in the following. The geometrical structure is in both cases the native zwitterionic conformation, obtained by NMR measurements as deposited in the protein data base. 73 For our gas phase simulations we consider the models to be charge neutral in total. To this end basic and acidic side chains have been restored to their neutral form by appropriate protonation or deprotonation The carboxyl and amino terminals were kept oppositely charged. A ribbon representation of both proteins is given in Figure 6 .

The LC-DFT and RHF calculations have been performed with the parallel version of NWCHEM6.3 on 8 CPUs, whereas LC-DFTB was run on a single core. As can be seen from the Table 4 a

$\dagger$ The geometry of the affected functional groups was subsequently optimized at the DFTB level (electronic temperature $T=500 \mathrm{~K}$ ) leaving the rest of the molecule fixed. 


\begin{tabular}{lrrrrrr}
\hline \hline & \multicolumn{3}{c}{ Chignolin } & \multicolumn{4}{c}{ Trp-cage } \\
& HOMO & LUMO & Gap & HOMO & LUMO & Gap \\
\hline LC-DFTB $(\omega=0.3)$ & -3.15 & 0.63 & 3.78 & -3.66 & -1.18 & 2.48 \\
LC-DFTB $(\omega \rightarrow \infty)$ & -5.90 & 2.14 & 8.04 & -6.14 & 1.32 & 7.46 \\
LC-PBE $/ 3-21 G$ & -3.78 & 0.34 & 4.12 & -4.46 & -1.72 & 2.74 \\
RHF $/ 3-21 G$ & -5.42 & 1.81 & 7.23 & -6.12 & -0.13 & 5.99 \\
\hline \hline & time [sec] & & time [sec] \\
\hline LC-DFTB $(\omega=0.3)$ & & 73 & & 882 \\
LC-DFTB $(\omega \rightarrow \infty)$ & & 78 & & 331 & \\
LC-PBE $/ 3-21 G$ & & 8841 & & 36844 & \\
RHF $/ 3-21 G$ & & 1577 & & 7534 & \\
\hline \hline
\end{tabular}

Table 4: Frontier orbital energies and fundamental gap (all in $\mathrm{eV}$ ) of the chignolin and Trp-cage zwitterions in the gas-phase for different theories.

good agreement in the description of the frontier orbital energies between full DFT and LC-DFTB is achieved. The timings can be found in the bottom part of the Table. We also performed LC-DFTB calculations for different values of the range-separation parameter $\omega$. The convergence (number of SCF cycles) for both proteins is depicted in the figure 6. As can be clearly seen, the convergence issue does not occur for typical values of the range-separation parameter. As the band gap opens, convergence generally improves.

These results indicate that LC-DFTB allows for reliable studies of proteins in the gas-phase both in the neutral and zwitterionic conformation. Certainly, further studies need to address also the energetics of the various conformers but already at this point LC-DFTB seems to be a useful tool for biological applications. In this context it might serve as the underlying electronic structure method for the fragment molecular orbital approach (FMO) ${ }^{78+80}$ which enables the quantum chemical study of biological systems with many thousands of atoms.

\section{CONCLUSION AND OUTLOOK}

In the present paper we addressed the implementation and benchmark of a long-range corrected exchange-correlation functional in the DFTB method, which we denote as LC-DFTB, for closedshell systems. The practical implementation requires the extension of tools for the evaluation of Slater-Koster parameters as well as some rather minor changes to the DFTB Hamiltonian. Thus the scheme can be easily implemented in existing ab initio software packages, which in principle contain all necessary routines for the integral evaluation and Hamiltonian construction. 
The Hamiltonian construction exhibits quadratic scaling and dominates the computational time for the systems tested. For larger systems the method is expected to show cubic scaling due to the diagonalization. The performance benefit with respect to first principles DFT with long-range corrected functionals still remains two to three orders of magnitude depending on the size of the basis set.

LC-DFTB shows clear signatures of correction of the delocalization problem in local DFT that is attributed to the self-interaction error. This has been demonstrated for the frontier orbitals of a set of organic molecules, where in general a promising agreement with full LC-DFT with double zeta basis (BNL/3-21G) has been observed. Remaining flaws were related to the minimal basis set characteristic for DFTB, which influences especially electron affinity levels. As a second example we calculated the static longitudinal polarizability of polyacetylene chains and provided evidence for the qualitatively correct description of the response potential.

The parametrization of the repulsive potential, which requires an adjustment due to the modified Hamiltonian, is presently on the way. With this development, thermochemical and structural properties may be investigated in addition to the electronic structure. The extension of the method to a spin-unrestricted formalism is likewise promising, since it would allow for the non-empirical tuning of the range-separation parameter ${ }^{22}$ Finally we would like to point out that the presented formalism is not restricted to the specific long-range corrected functional used in this study. The scheme can be easily adapted to conventional hybrid functionals like B3LYP or to screened exchange functionals like HSE,, 81 which are recently becoming increasingly popular for periodic systems and solid state applications.

\section{ACKNOWLEDGEMENT}

Financial support from the Deutsche Forschungsgemeinschaft (GRK 1570) is gratefully acknowledged. We thank Stephan Irle and Yoshio Nishimoto (Nagoya University) for making the authors aware of the potential applications of the presented method in the field of biological systems.

\section{APPENDIX A. NOTE ON THE NUMERICAL EVALUATION OF INTEGRALS}

The evaluation of electron repulsion integrals for Slater type orbitals (STO) as used in the DFTB method is a numerically challenging task. For the Coulomb interaction several algorithms based on the analytical expansions of STOs around a center are available. Recently, Seth and Ziegler ${ }^{32}$ extended these techniques to the case of a Yukawa interaction $(\exp (-\omega r) / r)$. In the present method the run-time evaluation of integrals is explicitly avoided by invoking the Slater-Koster scheme with 
precomputed parameters. Thus the integral evaluation does not affect the computational performance. We therefore resort to a simple, yet robust, numerical integration scheme proposed by Becke. 82 This scheme was designed for the evaluation of two-electron integrals over numerically defined charge distributions with Coulomb interaction. The modification for the case of Yukawa interaction is straightforward and laid out below.

The double integral over four arbitrary orbitals $\phi_{i}(\mathbf{r})$, which are in general located at different centers, and some interaction $g\left(\left|\mathbf{r}-\mathbf{r}^{\prime}\right|\right)$

$$
(a b \mid c d)=\iint \phi_{a}(\mathbf{r}) \phi_{b}(\mathbf{r}) g\left(\left|\mathbf{r}-\mathbf{r}^{\prime}\right|\right) \phi_{c}\left(\mathbf{r}^{\prime}\right) \phi_{d}\left(\mathbf{r}^{\prime}\right) d \mathbf{r} d \mathbf{r}^{\prime}
$$

can be seen as a special case of the integral over general charge distributions $\rho(\mathbf{r}), \sigma(\mathbf{r})$

$$
I=\iint \rho(\mathbf{r}) \sigma\left(\mathbf{r}^{\prime}\right) g\left(\left|\mathbf{r}-\mathbf{r}^{\prime}\right|\right) d \mathbf{r} d \mathbf{r}^{\prime}=\int \rho(\mathbf{r}) \underbrace{\left[\int \sigma\left(\mathbf{r}^{\prime}\right) g\left(\left|\mathbf{r}-\mathbf{r}^{\prime}\right|\right) d \mathbf{r}^{\prime}\right]}_{V(\mathbf{r})} d \mathbf{r} .
$$

The potential $V(\mathbf{r})$ can be obtained by solving the following boundary value problem with $V(\mathbf{r}) \rightarrow$ $0,|\mathbf{r}| \rightarrow \infty$

$$
\mathcal{D} V(\mathbf{r})=-4 \pi \sigma(\mathbf{r})
$$

with some differential operator $\mathcal{D}$. The function $g\left(\left|\mathbf{r}-\mathbf{r}^{\prime}\right|\right)$ in eq. 36 is the Greens function of the problem

$$
\mathcal{D} g\left(\left|\mathbf{r}-\mathbf{r}^{\prime}\right|\right)=-4 \pi \delta\left(\mathbf{r}-\mathbf{r}^{\prime}\right)
$$

The differential operator for the Coulomb interaction $g(r)=1 / r$ is $\mathcal{D}=\nabla^{2}$. The Yukawa interaction $g(r)=\exp (-\omega r) / r$ is the Greens function for the modified Helmholtz operator $\mathcal{D}=\nabla^{2}-\omega^{2}$. Thus the Poisson equation in the original work by Becke has to be replaced by the modified Helmholtz equation.

To make use of efficient quadrature schemes, Becke proposed a space partitioning and decomposition of the charge distribution into atom-centered portions. The space is partitioned by some set of functions $f_{A}(\mathbf{r})$, with $\sum_{A} f_{A}(\mathbf{r})=1, \forall \mathbf{r} \in \mathbb{R}^{3}$. Using this set of functions the density is divided into the atomic contributions

$$
\rho(\mathbf{r})=\sum_{A} f_{A}(\mathbf{r}) \rho(\mathbf{r})=\sum_{A} \rho_{A}(\mathbf{r})
$$

\footnotetext{
$\ddagger$ The choice of the numerical integrator can be additionally motivated by the fact that the DFTB method does in principle not rely on a specific type of orbitals as basis functions. This allows for more flexibility, especially in the context of atomic DFT calculations with converged numerical orbitals.
} 
This allows to write the integral in Eq. 36 as a sum of two-center integrals over atom-centered charge distributions $\rho_{A}, \sigma_{B}$

$$
I=\sum_{A B} I_{A B}=\sum_{A B} \iint \rho_{A}(\mathbf{r}) \sigma_{B}\left(\mathbf{r}^{\prime}\right) g\left(\left|\mathbf{r}-\mathbf{r}^{\prime}\right|\right) d \mathbf{r} d \mathbf{r}^{\prime}
$$

The inner part of the two-center integral $I_{A B}$ is evaluated as follows. In the Helmholtz equation

$$
\left(\nabla^{2}-\omega^{2}\right) V_{A}(\mathbf{r})=-4 \pi \rho_{A}(\mathbf{r})
$$

the potential and density are expanded into spherical harmonics

$$
\begin{aligned}
& V_{A}(\mathbf{r})=\sum_{l m} r^{-1} V_{A, l m}(r) Y_{l m}(\Omega) \\
& \rho_{A}(\mathbf{r})=\sum_{l m} \rho_{A, l m}(r) Y_{l m}(\Omega)
\end{aligned}
$$

Inserting these expansions into Eq. 41 gives a set of one-dimensional radial equations

$$
\left[\frac{d^{2}}{d r^{2}}-\frac{l(l+1)}{r^{2}}-\omega^{2}\right] V_{A, l m}(r)=-4 \pi r \rho_{A, l m}(r),
$$

with boundary conditions $\lim _{r \rightarrow 0} V_{A, l m}(r)=0, \lim _{r \rightarrow \infty} V_{A, l m}(r)=0$. These equations are solved using a finite difference method and from the resulting $V_{A, l m}$ the potential $V_{A}(\mathbf{r})$ is assembled according to Eq. 42. The remainder of the integration is performed on a two-center grid as described in the original paper by Becke.

\section{APPENDIX B. ANALYTICAL EVALUATION OF THE LONG-RANGE $\gamma$-INTEGRAL}

The $\gamma$-integral over the Yukawa interaction can be reduced to a one-dimensional integration 16

$$
\begin{aligned}
\gamma_{A B}^{\mathrm{Y}, \omega}\left(R_{A B}\right) & =\frac{\tau_{A}^{3} \tau_{B}^{3}}{(8 \pi)^{2}} \iint e^{-\tau_{A}\left|\mathbf{r}_{1}-\mathbf{R}_{A}\right|} \frac{e^{-\omega\left|\mathbf{r}_{1}-\mathbf{r}_{2}\right|}}{\left|\mathbf{r}_{1}-\mathbf{r}_{2}\right|} e^{-\tau_{B}\left|\mathbf{r}_{2}-\mathbf{R}_{B}\right|} d \mathbf{r}_{1} d \mathbf{r}_{2} \\
& =\frac{2 \tau_{A}^{4} \tau_{B}^{4}}{\pi R_{A B}} \int_{0}^{\infty} \frac{q \sin \left(q R_{A B}\right)}{\left(q^{2}+\tau_{A}^{2}\right)^{2}\left(q^{2}+\tau_{B}^{2}\right)^{2}\left(q^{2}+\omega^{2}\right)} d q
\end{aligned}
$$


where $R_{A B}=\left|\mathbf{R}_{A}-\mathbf{R}_{B}\right|$. This integral can now be further evaluated using the residue theorem. We obtain then the analytical expression ${ }^{\S}$

$$
\begin{aligned}
\gamma_{A B}^{\mathrm{Y}, \omega}\left(R_{A B}\right) & =\frac{\tau_{A}^{4} \tau_{B}^{4}}{\left(\tau_{A}^{2}-\omega^{2}\right)^{2}\left(\tau_{B}^{2}-\omega^{2}\right)^{2}} \frac{e^{-\omega R_{A B}}}{R_{A B}} \\
& -\left[e^{-\tau_{A} R_{A B}}\left(\frac{\tau_{A}^{2}}{\tau_{A}^{2}-\omega^{2}} \frac{\tau_{A} \tau_{B}^{4}}{2\left(\tau_{B}^{2}-\tau_{A}^{2}\right)^{2}}-\frac{\tau_{A}^{4}}{\left(\omega^{2}-\tau_{A}^{2}\right)^{2}} \frac{\left(\tau_{B}^{6}+3 \tau_{A}^{2} \tau_{B}^{4}+2 \omega^{2} \tau_{B}^{4}\right)}{\left(\tau_{A}^{2}-\tau_{B}^{2}\right)^{3} R_{A B}}\right)\right. \\
& \left.+e^{-\tau_{B} R_{A B}}\left(\frac{\tau_{B}^{2}}{\tau_{B}^{2}-\omega^{2}} \frac{\tau_{B} \tau_{A}^{4}}{2\left(\tau_{A}^{2}-\tau_{B}^{2}\right)^{2}}-\frac{\tau_{B}^{4}}{\left(\omega^{2}-\tau_{B}^{2}\right)^{2}} \frac{\left(\tau_{A}^{6}+3 \tau_{B}^{2} \tau_{A}^{4}+2 \omega^{2} \tau_{A}^{4}\right)}{\left(\tau_{B}^{2}-\tau_{A}^{2}\right)^{3} R_{A B}}\right)\right] .
\end{aligned}
$$

In the limit $\omega \rightarrow 0$ the integral is taken over the Coulomb interaction. In this case our analytical formula reduces to the result of Elstner et al. ${ }^{33}$ The long-range $\gamma$-integral is then given by the difference $\gamma_{A B}^{\mathrm{lr}}=\gamma_{A B}^{\mathrm{Y}, 0}-\gamma_{A B}^{\mathrm{Y}, \omega}$.

To obtain the on-site value $\left(R_{A B} \rightarrow 0, \quad A=B\right)$, we go back to the one-dimensional integral Eq. 46. We expand the term $\sin \left(q R_{A A}\right)$ around zero and integrate again using the residue theorem

$$
\begin{aligned}
& \lim _{R_{A A} \rightarrow 0} \frac{2 \tau_{A}^{8}}{\pi R_{A A}} \int_{0}^{\infty} \frac{q \sin \left(q R_{A A}\right)}{\left(q^{2}+\tau_{A}^{2}\right)^{4}\left(q^{2}+\omega^{2}\right)} d q \\
& =\frac{2 \tau_{A}^{8}}{\pi} \int_{0}^{\infty} \frac{q^{2}}{\left(q^{2}+\tau_{A}^{2}\right)^{4}\left(q^{2}+\omega^{2}\right)} d q \\
& =\frac{\tau_{A}^{8}}{48\left(\tau_{A}^{2}-\omega^{2}\right)^{4}}\left[\frac{3\left(5 \tau_{A}^{6}+15 \tau_{A}^{4} \omega^{2}-5 \tau_{A}^{2} \omega^{4}+\omega^{6}\right)}{\tau_{A}^{5}}-48 \omega\right] .
\end{aligned}
$$

This yields the on-site value for the integral Eq. 21

$$
\gamma_{A A}^{\mathrm{lr}}=\frac{5}{16} \tau_{A}-\frac{\tau_{A}^{8}}{\left(\tau_{A}^{2}-\omega^{2}\right)^{4}}\left[\frac{5 \tau_{A}^{6}+15 \tau_{A}^{4} \omega^{2}-5 \tau_{A}^{2} \omega^{4}+\omega^{6}}{16 \tau_{A}^{5}}-\omega\right]
$$

\section{APPENDIX C. THE HUBBARD PARAMETER IN LC-DFTB}

We derive the expression for the Hubbard parameter of atomic LC-DFTB. Note that in this case $S_{\mu \nu}=\delta_{\mu \nu}, \gamma_{\mu \nu}^{\mathrm{fr} / \mathrm{Ir}}=\gamma_{A A}^{\mathrm{fr} / \mathrm{Ir}}$ and $H_{\mu \nu}^{0}=\delta_{\mu \nu} \epsilon_{\mu}$. Using the orhogonality condition $\sum_{\mu} c_{\mu, i} c_{\mu, j}=\delta_{i j}$ we obtain the total LC-DFTB energy of one atom in terms of occupation numbers $n_{i}$

$$
\begin{aligned}
E^{\mathrm{atom}} & =\sum_{\mu} P_{\mu \mu} \epsilon_{\mu}+\frac{1}{2} \gamma_{A A}^{\mathrm{fr}} \sum_{\mu \alpha} \Delta P_{\mu \mu} \Delta P_{\alpha \alpha}-\frac{1}{4} \gamma_{A A}^{\mathrm{lr}} \sum_{\mu \nu} \Delta P_{\mu \nu} \Delta P_{\mu \nu} \\
& =\frac{1}{2} \sum_{i j} n_{i} n_{j} \gamma_{A A}^{\mathrm{fr}}-\frac{1}{4} \sum_{i} n_{i}^{2} \gamma_{A A}^{\mathrm{lr}}+\text { terms linear in } n_{i}
\end{aligned}
$$

$\S$ The analytical formula Eq. 47 has been derived after the calculations for this work have been done. For the presented results the long-range integral has been evaluated with a numerical integrator. We note that the analytical formula is more practicable and is recommended for use in practical implementation of the method. 
We consider further only terms, quadratic in the occupation numbers, which belong to the highest occupied shell. It contains $n$ electrons, equally distributed over the shell. The occupation number for an orbital is then $n / d_{l}$, where $d_{l}=2 l+1$ is the degeneracy of the shell with angular momentum $l$. Then the energy can be written as

$$
\begin{aligned}
E^{\text {atom }} & =\frac{1}{2} \gamma_{A A}^{\mathrm{fr}} \sum_{i j}^{\text {shell }}\left(\frac{n}{d_{l}}\right)^{2}-\frac{1}{4} \gamma_{A A}^{\mathrm{lr}} \sum_{i}^{\text {shell }}\left(\frac{n}{d_{l}}\right)^{2}+\text { terms linear in } n \\
& =\frac{1}{2} \gamma_{A A}^{\mathrm{fr}} d_{l}^{2}\left(\frac{n}{d_{l}}\right)^{2}-\frac{1}{4} \gamma_{A A}^{\mathrm{lr}} d_{l}\left(\frac{n}{d_{l}}\right)^{2}+\text { terms linear in } n \\
& =\frac{1}{2} \gamma_{A A}^{\mathrm{fr}} n^{2}-\frac{1}{4} \gamma_{A A}^{\mathrm{lr}} \frac{n^{2}}{d_{l}}+\text { terms linear in } n
\end{aligned}
$$

Thus the second derivative with respect to the occupation numbers of the highest occupied shell is

$$
\frac{\partial^{2} E^{\mathrm{atom}}}{\partial n^{2}}=\gamma_{A A}^{\mathrm{fr}}-\frac{1}{2} \frac{1}{2 l+1} \gamma_{A A}^{\mathrm{lr}}
$$

And together with Eq. 51 the atomic Hubbard from the LC-DFTB is obtained

$$
U_{A}=\frac{5}{16} \tau\left[1-\frac{1}{2(2 l+1)}\left(1-\frac{\tau^{8}+3 \tau^{6} \omega^{2}-\tau^{4} \omega^{4}+0.2 \omega^{6} \tau^{2}-3.2 \tau^{7} \omega}{\left(\tau^{2}-\omega^{2}\right)^{4}}\right)\right]
$$

This equation defines the decay constant $\tau$.

\section{REFERENCES}

1. K. Burke, "Perspective on density functional theory," J. Chem. Phys., vol. 136, no. 15, p. 150901, 2012.

2. D. Bowler, T. Miyazaki, and M. Gillan, "Recent progress in linear scaling ab initio electronic structure techniques," J. Phys.: Condens. Matter, vol. 14, no. 11, p. 2781, 2002.

3. X. D. Xiang, X. Sun, G. Briceno, Y. Lou, K.-A. Wang, H. Chang, W. G. Wallace-Freedman, S.W. Chen, and P. G. Schultz, "A combinatorial approach to materials discovery," Science, vol. 268, no. 5218, pp. 1738-1740, 1995.

4. A. Jain, S. P. Ong, G. Hautier, W. Chen, W. D. Richards, S. Dacek, S. Cholia, D. Gunter, D. Skinner, G. Ceder, and K. a. Persson, "The Materials Project: A materials genome approach to accelerating materials innovation," APL Materials, vol. 1, no. 1, p. 011002, 2013.

5. M. Elstner and G. Seifert, "Density functional tight binding," Philosophical Transactions of the Royal Society A: Mathematical, Physical and Engineering Sciences, vol. 372, no. 2011, p. 20120483, 2014.

6. Q. Cui and M. Elstner, "Density functional tight binding: values of semi-empirical methods in an ab initio era," Phys. Chem. Chem. Phys., vol. 16, no. 28, pp. 14368-14377, 2014. 
7. C. Köhler, T. Frauenheim, B. Hourahine, G. Seifert, and M. Sternberg, "Treatment of collinear and noncollinear electron spin within an approximate density functional based method," J. Phys. Chem. A, vol. 111, no. 26, pp. 5622-5629, 2007.

8. M. Elstner, P. Hobza, T. Frauenheim, S. Suhai, and E. Kaxiras, "Hydrogen bonding and stacking interactions of nucleic acid base pairs: a density-functional-theory based treatment," J. Chem. Phys., vol. 114, no. 12, pp. 5149-5155, 2001.

9. W.-G. Han, M. Elstner, K. Jalkanen, T. Frauenheim, and S. Suhai, "Hybrid scc-dftb/molecular mechanical studies of h-bonded systems and of n-acetyl-(I-ala) nn-methylamide helices in water solution," Int. J. Quantum Chem., vol. 78, no. 6, pp. 459-479, 2000.

10. Q. Cui, M. Elstner, E. Kaxiras, T. Frauenheim, and M. Karplus, "A qm/mm implementation of the self-consistent charge density functional tight binding (scc-dftb) method," J. Phys. Chem. B, vol. 105, no. 2, pp. 569-585, 2001.

11. T. A. Niehaus, S. Suhai, F. Della Sala, P. Lugli, M. Elstner, G. Seifert, and T. Frauenheim, "Tightbinding approach to time-dependent density-functional response theory," Phys. Rev. B, vol. 63, p. 085108, Feb 2001.

12. T. A. Niehaus, "Approximate time-dependent density functional theory," J. Mol. Struct.: THEOCHEM, vol. 914, p. 38, 2009.

13. A. Dominguez, B. Aradi, T. Frauenheim, V. Lutsker, and T. A. Niehaus, "Extensions of the timedependent density functional based tight-binding approach," J. Chem. Theory Comput., vol. 9, no. 11, pp. 4901-4914, 2013.

14. R. Rüger, E. van Lenthe, Y. Lu, J. Frenzel, T. Heine, and L. Visscher, "Efficient calculation of electronic absorption spectra by means of intensity-selected time-dependent density functional tight binding," J. Chem. Theory Comput., vol. 11, no. 1, pp. 157-167, 2014.

15. J. C. Slater and G. F. Koster, "Simplified Icao method for the periodic potential problem," Phys. Rev., vol. 94, no. 6, pp. 1498-1524, 1954.

16. T. Niehaus and F. Della Sala, "Range separated functionals in the density functional based tight-binding method: Formalism," physica status solidi (b), vol. 249, p. 237, 2012.

17. P. M. W. Gill, R. D. Adamason, and J. A. Pople, "Coulomb-attenuated exchange energy density functionals," Molecular Physics, vol. 88, no. 4, pp. 1005-1009, 1996.

18. A. Savin, Recent Developments and Applications of Modern Density Functional Theory, pp. 327-357. Amsterdam: Elsevier, 1996.

19. T. Leininger, H. Stoll, H.-J. Werner, and A. Savin, "Combining long-range configuration interaction with short-range density functionals," Chem. Phys. Lett., vol. 275, p. 151, 1997.

20. T. Yanai, D. P. Tew, and N. C. Handy, "A new hybrid exchangecorrelation functional using the coulomb-attenuating method (cam-b3lyp)," Chemical Physics Letters, vol. 393, no. 1-3, pp. 51-57, 2004. 
21. H. likura, T. Tsuneda, T. Yanai, and K. Hirao, "A long-range correction scheme for generalizedgradient-approximation exchange functionals," J. Chem. Phys., vol. 115, p. 3540, 2001.

22. R. Baer, E. Livshits, and U. Salzner, "Tuned range-separated hybrids in density functional theory," Annu. Rev. Phys. Chem., vol. 61, pp. 85-109, 2010.

23. L. Kronik and S. Kümmel, "Gas-phase valence-electron photoemission spectroscopy using density functional theory," in Top Curr Chem, vol. 347, pp. 137-192, Springer-Verlag Berlin Heidelberg, 2014.

24. R. Baer and D. Neuhauser, "Density functional theory with correct long-range asymptotic behavior," Phys. Rev. Lett., vol. 94, p. 043002, Feb 2005.

25. E. Livshits and R. Baer, "A well-tempered density functional theory of electrons in molecules," Phys. Chem. Chem. Phys., vol. 9, pp. 2932-2941, 2007.

26. O. A. Vydrov and G. E. Scuseria, "Assessment of a long-range corrected hybrid functional," The Journal of Chemical Physics, vol. 125, no. 23, p. 234109, 2006.

27. M. A. Rohrdanz, K. M. Martins, and J. M. Herbert, "A long-range-corrected density functional that performs well for both ground-state properties and time-dependent density functional theory excitation energies, including charge-transfer excited states," The Journal of Chemical Physics, vol. 130, no. 5, p. 054112, 2009. defines the LRC-wPBEh functional.

28. Y. Akinaga and S. Ten-no, "Range-separation by the yukawa potential in long-range corrected density functional theory with gaussian-type basis functions," Chemical Physics Letters, vol. 462, no. 4-6, pp. 348-351, 2008.

29. J. P. Perdew, K. Burke, and M. Ernzerhof, "Generalized gradient approximation made simple [phys. rev. lett. 77, 3865 (1996)]," Phys. Rev. Lett., vol. 78, pp. 1396-1396, Feb 1997.

30. J. E. Robinson, F. Bassani, R. S. Knox, and J. R. Schrieffer, "Screening correction to the slater exchange potential," Phys. Rev. Lett., vol. 9, pp. 215-217, Sep 1962.

31. A. Savin and H.-J. Flad, "Density functionals for the Yukawa electron-electron interaction," International Journal of Quantum Chemistry, vol. 56, no. 4, pp. 327-332, 1995.

32. M. Seth and T. Ziegler, "Range-Separated Exchange Functionals with Slater-Type Functions," Journal of Chemical Theory and Computation, vol. 8, no. 3, pp. 901-907, 2012.

33. M. Elstner, D. Porezag, G. Jungnickel, J. Elsner, M. Haugk, T. Frauenheim, S. Suhai, and G. Seifert, "Self-consistent-charge density-functional tight-binding method for simulations of complex materials properties," Phys. Rev. B, vol. 58, pp. 7260-7268, Sep 1998.

34. T. A. Niehaus, M. Elstner, T. Frauenheim, and S. Suhai, "Application of an approximate densityfunctional method to sulfur containing compounds," J. Mol. Struct. - Theochem, vol. 541, pp. 185194, May 2001.

35. M. Häser and R. Ahlrichs, "Improvements on the direct SCF method," J. Comput. Chem., vol. 10, no. 1, pp. 104-111, 1989. 
36. B. Aradi, B. Hourahine, and T. Frauenheim, "Dftb+, a sparse matrix-based implementation of the dftb method," J. Phys. Chem. A, vol. 111, no. 26, pp. 5678-5684, 2007.

37. S. Refaely-Abramson, S. Sharifzadeh, N. Govind, J. Autschbach, J. B. Neaton, R. Baer, and L. Kronik, "Quasiparticle spectra from a nonempirical optimally tuned range-separated hybrid density functional," Phys. Rev. Lett., vol. 109, p. 226405, Nov 2012.

38. A. J. Cohen, P. Mori-Sanchez, and W. Yang, "Challenges for Density Functional Theory," Chemical Reviews, vol. 112, no. 1, pp. 289-320, 2012. PMID: 22191548.

39. A. J. Cohen, P. Mori-Sanchez, and W. Yang, "Fractional charge perspective on the band gap in density-functional theory," Phys. Rev. B, vol. 77, p. 115123, Mar 2008.

40. A. Seidl, A. Görling, P. Vogl, J. A. Majewski, and M. Levy, "Generalized kohn-sham schemes and the band-gap problem," Phys. Rev. B, vol. 53, pp. 3764-3774, Feb 1996.

41. J. P. Perdew, "Density functional theory and the band gap problem," International Journal of Quantum Chemistry, vol. 28, no. S19, pp. 497-523, 1985.

42. X. Blase, C. Attaccalite, and V. Olevano, "First-principles $G W$ calculations for fullerenes, porphyrins, phtalocyanine, and other molecules of interest for organic photovoltaic applications," Phys. Rev. B, vol. 83, p. 115103, Mar 2011.

43. L. Kronik, T. Stein, S. Refaely-Abramson, and R. Baer, "Excitation Gaps of Finite-Sized Systems from Optimally Tuned Range-Separated Hybrid Functionals," Journal of Chemical Theory and Computation, vol. 8, no. 5, pp. 1515-1531, 2012.

44. S. Refaely-Abramson, R. Baer, and L. Kronik, "Fundamental and excitation gaps in molecules of relevance for organic photovoltaics from an optimally tuned range-separated hybrid functional," Phys. Rev. B, vol. 84, p. 075144, Aug 2011.

45. P. Mori-Sanchez, P.nchez, A. J. Cohen, and W. Yang, "Many-electron self-interaction error in approximate density functionals," The Journal of Chemical Physics, vol. 125, no. 20, p. 201102, 2006.

46. T. Körzdörfer, R. M. Parrish, N. Marom, J. S. Sears, C. D. Sherrill, and J.-L. Brédas, "Assessment of the performance of tuned range-separated hybrid density functionals in predicting accurate quasiparticle spectra," Phys. Rev. B, vol. 86, p. 205110, Nov 2012.

47. A. D. Becke, "Densityfunctional thermochemistry. III. The role of exact exchange," The Journal of Chemical Physics, vol. 98, no. 7, pp. 5648-5652, 1993.

48. C. Lee, W. Yang, and R. G. Parr, "Development of the colle-salvetti correlation-energy formula into a functional of the electron density," Phys. Rev. B, vol. 37, pp. 785-789, Jan 1988.

49. S. H. Vosko, L. Wilk, and M. Nusair, "Accurate spin-dependent electron liquid correlation energies for local spin density calculations: a critical analysis," Canadian Journal of Physics, vol. 58, no. 8, pp. 1200-1211, 1980. 
50. P. J. Stephens, F. J. Devlin, C. F. Chabalowski, and M. J. Frisch, "Ab Initio Calculation of Vibrational Absorption and Circular Dichroism Spectra Using Density Functional Force Fields," The Journal of Physical Chemistry, vol. 98, no. 45, pp. 11623-11627, 1994.

51. M. Valiev, E. J. Bylaska, N. Govind, K. Kowalski, T. P. Straatsma, H. J. Van Dam, D. Wang, J. Nieplocha, E. Apra, T. L. Windus, and W. A. de Jong, "Nwchem: a comprehensive and scalable open-source solution for large scale molecular simulations," Comput. Phys. Commun., vol. 181, no. 9, pp. 1477-1489, 2010.

52. P. Linstrom and W. Mallard, NIST Chemistry WebBook, NIST Standard Reference Database Number 69. National Institute of Standards and Technology.

53. N. Dori, M. Menon, L. Kilian, M. Sokolowski, L. Kronik, and E. Umbach, "Valence electronic structure of gas-phase 3,4,9,10-perylene tetracarboxylic acid dianhydride: Experiment and theory," Phys. Rev. $B$, vol. 73, p. 195208, May 2006.

54. N. Marom, F. Caruso, X. Ren, O. T. Hofmann, T. Körzdörfer, J. R. Chelikowsky, A. Rubio, M. Scheffler, and P. Rinke, "Benchmark of $g w$ methods for azabenzenes," Phys. Rev. B, vol. 86, p. 245127, Dec 2012.

55. S. Sharifzadeh, A. Biller, L. Kronik, and J. B. Neaton, "Quasiparticle and optical spectroscopy of the organic semiconductors pentacene and ptcda from first principles," Phys. Rev. B, vol. 85, p. 125307, Mar 2012.

56. J. Sauther, J. Wüsten, S. Lach, and C. Ziegler, "Gas phase and bulk ultraviolet photoemission spectroscopy of 3,4,9,10-perylene-tetracarboxylic dianhydride, 1,4,5,8-naphthalene-tetracarboxylic dianhydride, and 1,8-naphthalene-dicarboxylic anhydride," The Journal of Chemical Physics, vol. 131, no. 3, p. $034711,2009$.

57. X. Gonze, P. Ghosez, and R. W. Godby, "Density-polarization functional theory of the response of a periodic insulating solid to an electric field," Phys. Rev. Lett., vol. 74, pp. 4035-4038, May 1995.

58. S. J. A. van Gisbergen, P. R. T. Schipper, O. V. Gritsenko, E. J. Baerends, J. G. Snijders, B. Champagne, and B. Kirtman, "Electric field dependence of the exchange-correlation potential in molecular chains," Phys. Rev. Lett., vol. 83, pp. 694-697, Jul 1999.

59. A. Karolewski, R. Armiento, and S. Kümmel, "Polarizabilities of Polyacetylene from a FieldCounteracting Semilocal Functional," Journal of Chemical Theory and Computation, vol. 5, no. 4, pp. 712-718, 2009.

60. B. Champagne, E. A. Perpete, S. J. A. van Gisbergen, E.-J. Baerends, J. G. Snijders, C. Soubra-Ghaoui, K. A. Robins, and B. Kirtman, "Assessment of conventional density functional schemes for computing the polarizabilities and hyperpolarizabilities of conjugated oligomers: An ab initio investigation of polyacetylene chains," The Journal of Chemical Physics, vol. 109, no. 23, pp. 10489-10498, 1998.

61. S. M. Lindsay and M. A. Ratner, "Molecular transport junctions: Clearing mists," Advanced Materials, vol. 19, no. 1, pp. 23-31, 2007. 
62. H. Sekino, Y. Maeda, M. Kamiya, and K. Hirao, "Polarizability and second hyperpolarizability evaluation of long molecules by the density functional theory with long-range correction," The Journal of Chemical Physics, vol. 126, no. 1, p. 014107, 2007.

63. S. Kaminski, T. J. Giese, M. Gaus, D. M. York, and M. Elstner, "Extended Polarization in Third-Order SCC-DFTB from Chemical-Potential Equalization," The Journal of Physical Chemistry A, vol. 116, no. 36, pp. 9131-9141, 2012. PMID: 22894819.

64. S. Kaminski, M. Gaus, and M. Elstner, "Improved Electronic Properties from Third-Order SCC-DFTB with Cost Efficient Post-SCF Extensions," The Journal of Physical Chemistry A, vol. 116, no. 48, pp. 11927-11937, 2012. PMID: 23167841.

65. K. B. Shelimov, D. E. Clemmer, R. R. Hudgins, and M. F. Jarrold, "Protein Structure in Vacuo: Gas-Phase Conformations of BPTI and Cytochrome c," Journal of the American Chemical Society, vol. 119, no. 9, pp. 2240-2248, 1997.

66. J. Fenn, M. Mann, C. Meng, S. Wong, and C. Whitehouse, "Electrospray ionization for mass spectrometry of large biomolecules," Science, vol. 246, no. 4926, pp. 64-71, 1989.

67. H. N. Chapman, A. Barty, M. J. Bogan, S. Boutet, M. Frank, S. P. Hau-Riege, S. Marchesini, B. W. Woods, S. Bajt, and W. H. Benner, "Femtosecond diffractive imaging with a soft-x-ray free-electron laser," Nature Physics, vol. 2, pp. 839-843, 2006.

68. T. Meyer, V. Gabelica, H. Grubmüller, and M. Orozco, "Proteins in the gas phase," Wiley Interdisciplinary Reviews: Computational Molecular Science, vol. 3, no. 4, pp. 408-425, 2013.

69. R. Suenram and F. Lovas, "Millimeter wave spectrum of glycine. a new conformer," J. Am. Chem. Soc., vol. 102, no. 24, pp. 7180-7184, 1980.

70. W. D. Price, R. A. Jockusch, and E. R. Williams, "Is arginine a zwitterion in the gas phase?," J. Am. Chem. Soc., vol. 119, no. 49, pp. 11988-11989, 1997.

71. R. Marchese, R. Grandori, P. Carloni, and S. Raugei, "On the zwitterionic nature of gas-phase peptides and protein ions," PLoS Comput. Biol., vol. 6, p. e1000775, 2010.

72. Y. Nishimoto and S. Irle private communication.

73. S. Honda, K. Yamasaki, Y. Sawada, and H. Morii, "10 Residue Folded Peptide Designed by Segment Statistics," Structure, vol. 12, no. 8, pp. 1507-1518, 2004.

74. Neidigh Jonathan W., Fesinmeyer R. Matthew, and Andersen Niels H., "Designing a 20-residue protein," Nat Struct Mol Biol, vol. 9, pp. 425-430, jun 2002. 10.1038/nsb798.

75. A. Patriksson, C. M. Adams, F. Kjeldsen, R. A. Zubarev, and D. van der Spoel, "A Direct Comparison of Protein Structure in the Gas and Solution Phase: The Trp-cage," The Journal of Physical Chemistry B, vol. 111, no. 46, pp. 13147-13150, 2007. PMID: 17973523.

76. F. Kjeldsen, O. A. Silivra, and R. A. Zubarev, "Zwitterionic States in Gas-Phase Polypeptide lons Revealed by 157-nm Ultra-Violet Photodissociation," Chemistry - A European Journal, vol. 12, no. 30, pp. 7920-7928, 2006. 
77. W. Humphrey, A. Dalke, and K. Schulten, "VMD - Visual Molecular Dynamics," Journal of Molecular Graphics, vol. 14, pp. 33-38, 1996.

78. D. G. Fedorov, T. Nagata, and K. Kitaura, "Exploring chemistry with the fragment molecular orbital method," Phys. Chem. Chem. Phys., vol. 14, pp. 7562-7577, 2012.

79. T. Nagata, K. Brorsen, D. G. Fedorov, K. K. , and M. S. G. , "Fully analytic energy gradient in the fragment molecular orbital method," The Journal of Chemical Physics, vol. 134, no. 12, p. 124115, 2011.

80. Y. Nishimoto, D. G. Fedorov, and S. Irle, "Density-Functional Tight-Binding Combined with the Fragment Molecular Orbital Method," Journal of Chemical Theory and Computation, vol. 10, no. 11, pp. 4801-4812, 2014.

81. J. Heyd, G. Scuseria, and M. Ernzerhof, "Hybrid functionals based on a screened coulomb potential," J. Chem. Phys., vol. 118, p. 8207, 2003.

82. A. D. Becke and R. M. Dickson, "Numerical solution of poisson's equation in polyatomic molecules," The Journal of Chemical Physics, vol. 89, no. 5, pp. 2993-2997, 1988. 


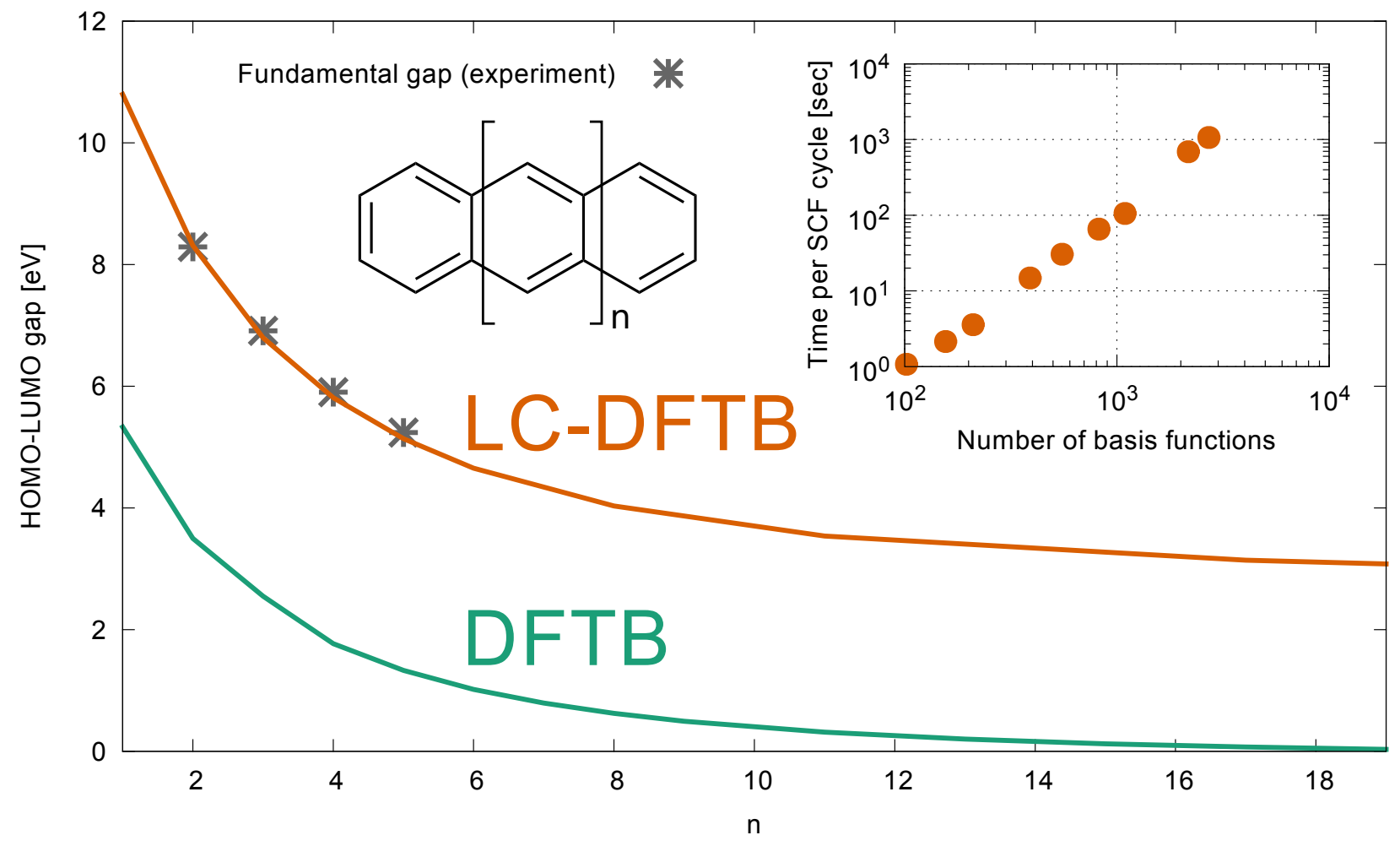

Figure 7: Table of Contents Graphic 